\title{
EQUIVALENCE CLASSES OF MAXIMAL ORDERS
}

\author{
SUSAN WILLIAMSON
}

Introduction. Let $k$ denote the quotient field of a complete discrete rank one valuation ring $R$. The purpose of this paper is to establish a relationship between the Brauer group of $k$ and the set of maximal orders over $R$ which are equivalent to crossed products over tamely ramified extensions of $R$.

The Brauer group $B(k)$ of $k$ is the union of groups $H^{2}(G, U(L))$ where $L$ ranges over the set of all finite Galois extensions of $k$ and $G$ denotes the Galois group of $L$ over $k$ (see pp. 206-207 of [2]). The subset $V(k)=$ $\cup H^{2}(G, U(L))$ where $L$ ranges over all unramified extensions of $k$ forms a subgroup of $B(k)$. In Section 1 we associate to each element of $V(k)$ a positive integer called its Brauer number. Then we define $T(k)$ to be the set of elements of $V(k)$ whose Brauer numbers are relatively prime to the characteristic of $\bar{R}$, and prove that $T(k)$ is a subgroup of $B(k)$. The object of the paper is to prove the following main theorem.

Theorem. Let $k$ denote the quotient field of a complete discrete rank one valuation ring $R . \quad A$ maximal order over $R$ in a central simple k-algebra $\Sigma$ is equivalent to a crossed product over a tamely ramified extension of $R$ if and only if the Brauer class of $\Sigma$ is in the subgroup $T(k)$ of $B(k)$.

The method of proof employs the theory of crossed products, and entails the construction of certain wildly ramified Galois extensions of $k$. For this, a separate treatment of the equicharacteristic case and the case of unequal characteristic is necessary (Sections 2 and 3 respectively).

We obtain as a corollary to the main theorem the fact that if $R$ is an equicharacteristic ring of characteristic zero, then every maximal $R$-order is equivalent to a crossed product over a tamely ramified extension of $R$. We then exhibit the existence of a maximal $R$-order which is not equivalent to a

Received November 18, 1966. 
crossed product in the case when $R$ is a complete discrete rank one valuation ring with perfect residue class field.

The following notation shall be in use throughout the paper. The multiplicative group of units of a ring $R$ shall be denoted by $U(R)$, and the radical of $R$ by $\operatorname{rad} R$. Unless otherwise stated, $R$ shall always denote a complete discrete rank one valuation ring, $\pi$ its prime element, and $k$ its quotient field. The definitions of crossed product and hereditary order may be found in [10]. For the definitions of tame and wild ramification we refer the reader to [9]. The definition of the $i^{t h}$ ramification group is given on p. 73 of [7].

For the convenience of the reader we define the notions of equivalence which shall be used in the paper. A pair of central simple algebras $\Sigma_{1}$ and $\Sigma_{2}$ over a field $k$ are said to be equivalent if there exist finite dimensional vector spaces $V_{1}$ and $V_{2}$ over $k$ together with a $k$-algebra isomorphism

$$
\Sigma_{1} \otimes_{R} \operatorname{Hom}_{k}\left(V_{1}, V_{1}\right) \approx \Sigma_{2} \otimes_{R} \operatorname{Hom}_{k}\left(V_{2}, V_{2}\right) \text {. }
$$

The set of equivalence classes of central simple algebras over a field $k$ forms an Abelian group called the Brauer group of $k$. The inverse of the equivalence class determined by the central simple algebra $\Sigma$ is the equivalence class determined by its opposite algebra $\Sigma^{0}$ (see Section 5 of [3]).

Let $R$ denote a discrete rank one valuation ring. The set of maximal orders $M^{\prime}(R)$ over $R$ forms a subset of the set $H^{\prime}(R)$ of hereditary orders over $R$ (see [4]). In [3] Auslander and Goldman have defined a pair of hereditary $R$-orders $\Lambda_{1}$ and $\Lambda_{2}$ to be equivalent if there exist finitely generated free $R$-modules $E_{1}$ and $E_{2}$ and an $R$-algebra isomorphism

$$
\Lambda_{1} \otimes_{R} \operatorname{Hom}_{R}\left(E_{1}, E_{1}\right) \approx \Lambda_{2} \otimes_{R} \operatorname{Hom}_{R}\left(E_{2}, E_{2}\right) .
$$

An hereditary order equivalent to a maximal order is itself a maximal order. The set of maximal orders in a fixed central simple algebra are isomorphic. Finally we mention that the equivalence relation on the set of maximal orders over $R$ is induced by the Brauer group of the quotient field $k$ of $R$. That is to say, if $\Sigma_{1}$ and $\Sigma_{2}$ are equivalent central simple algebras over the quotient field of a discrete rank one valuation ring, then the maximal orders of $\Sigma_{1}$ are equivalent to the maximal orders of $\Sigma_{2}$ (see Lemma 2.1 of [11]).

1. The Brauer number. Let $k$ denote the quotient field of a complete discrete rank one valuation ring $R$ and consider the subset $V(k)$ of the 
Brauer group $B(k)$ of $k$ defined by $V(k)=\cup H^{2}(G, U(L))$ where the union is taken over the set of all unramified Galois extensions $L$ of $k$. It is well known that $V(k)=B(k)$ when $\bar{R}$ is perfect. For an example to show that $V(k)$ need not equal $B(k)$ see Exer. 2 p. 224 of [7].

Proposition 1.1 The set $V(k)$ is a subgroup of the Brauer group of $k$.

Proof. Consider crossed products $\Sigma_{1}=\Delta\left(f_{1}, L_{1}, G_{1}\right)$ and $\Sigma_{2}=\Delta\left(f_{2}, L_{2}, G_{2}\right)$ where the $L_{i}$ are unramified Galois extensions of $k$ and $G_{i}$ denotes the Galois group of $L_{i}$ over $k$. In order to prove the proposition it suffices to show that the Brauer class of $\Delta\left(f_{1}, L_{1}, G_{1}\right) \otimes_{k} \Delta\left(f_{2}, L_{2}, G_{2}\right)^{0}$ is in $V(k)$ where $\Delta\left(f_{2}, L_{2}, G_{2}\right)^{0}$ denotes the opposite ring of $\Delta\left(f_{2}, L_{2}, G_{2}\right)$.

The compositum $L_{1} L_{2}$ of $L_{1}$ and $L_{2}$ is an unramified extension of $k$ according to Cor. 3-2-8 of [9]. For $i=1,2$ let $g_{i}$ denote the image of $f_{i}$ under the inflation map $Z^{2}\left(G_{i}, U\left(L_{i}\right)\right) \longrightarrow Z^{2}\left(G_{1} G_{2}, U\left(L_{1} L_{2}\right)\right)$ where $G_{1} G_{2}$ denotes the Galois group of $L_{1} L_{2}$ over $k$. It is well known that $\Delta\left(f_{i}, L_{i}, G_{i}\right)$ is equivalent to $\Delta\left(g_{2}, L_{1} L_{2}, G_{1} G_{2}\right)$ for $i=1,2$ (see for example Thm. $8.5 E$ of [1]). Therefore $\Sigma_{1} \otimes \Sigma_{2}^{0}$ is equivalent to $\Delta\left(g_{1}, L_{1} L_{2}, G_{1} G_{2}\right) \otimes \Delta\left(g_{2}^{-1}, L_{1} L_{2}, G_{1} G_{2}\right)$ since $\Delta\left(g_{2}^{-1}, L_{1} L_{2}, G_{1} G_{2}\right)$ represents the Brauer class of $\sum_{2}^{0}$. The fact that $\Delta\left(g_{1}, L_{1} L_{2}\right.$, $\left.G_{1} G_{2}\right) \otimes \Delta\left(g_{2}^{-1}, L_{1} L_{2}, G_{1} G_{2}\right)$ is equivalent to $\Delta\left(g_{1} g_{2}^{-1}, L_{1} L_{2}, G_{1} G_{2}\right)$ (see Thm. $8.5 \mathrm{~A}$ of [1] or pp. 404-405 of [3]) implies that the Brauer class of $\Sigma_{1} \otimes \Sigma_{2}^{0}$ is in $V(k)$.

For convenience of notation we shall always denote the Brauer class in $B(k)$ of a central simple $k$-algebra $\Sigma$ by $\widetilde{\Sigma}$. We proceed to define the Brauer number of an element of $V(k)$. A central simple $k$-algebra $\sum$ for which $\widetilde{\Sigma}$ is in $V(k)$ is equivalent to a crossed product $\Delta(f, L, G)$ for some unramified Galois extension $L$ of $k$ with Galois group $G$. Let $S$ denote the integral closure of $R$ in $L$ and consider the exact sequence of cohomology groups

$$
(1) \longrightarrow H^{2}(G, U(S)) \longrightarrow H^{2}(G, U(L)) \stackrel{\phi}{\longrightarrow} H^{2}\left(G, Z^{+}\right) \longrightarrow(1)
$$

defined explicitly on p. 193 of [7].

Definition. The Brauer number of an element $\widetilde{\Sigma}$ of $V(k)$ is defined to be the order of the image of the cohomology class $[f]$ in $H^{2}\left(G, Z^{+}\right)$under the $\operatorname{map} \phi$. 
Observe that the Brauer number of $\widetilde{\Sigma}$ is the least positive integer $n$ such that $\left[f^{n}\right]$ is in the image of the natural map $H^{2}(G, U(S)) \longrightarrow H^{2}(G, U(L))$. Therefore when the Brauer number of $\widetilde{\Sigma}$ is 1 , we know by Thm. 2. 3 of [11] that a maximal order in $\Sigma$ is equivalent to a crossed product over a tamely ramified extension of $R$.

The Brauer number is well defined according to the next proposition.

Proposition 1.2. The Brauer number of an element $\widetilde{\Sigma}$ of $V(k)$ is independent of the choice of representative of $\widetilde{\Sigma}$ as a crossed product over an unramified extension of $k$.

Proof. Let $\Delta\left(f_{1}, L_{1}, G_{1}\right)$ and $\Delta\left(f_{2}, L_{2}, G_{2}\right)$ denote equivalent central simple $k$-algebras, where $L_{1}$ and $L_{2}$ are unramified Galois extensions of $k$ with Galois groups $G_{1}$ and $G_{2}$ respectively. Let $L_{1} L_{2}$ denote the compositum of $L_{1}$ and $L_{2}$, and $G_{1} G_{2}$ the Galois group of $L_{1} L_{2}$ over $k$. Observe that $L_{1} L_{2}$ is an unramified extension of $k$. For $i=1,2$ let $\left[g_{i}\right]$ denote the image of $\left[f_{i}\right]$ under the inflation map $H^{2}\left(G_{i}, U\left(L_{i}\right)\right) \longrightarrow H^{2}\left(G_{1} G_{2}, U\left(L_{1} L_{2}\right)\right)$. The assumption that $\Delta\left(f_{1}, L_{1}, G_{1}\right)$ is equivalent to $\Delta\left(f_{2}, L_{2}, G_{2}\right)$ implies that $\left[g_{1}\right]=\left[g_{2}\right]$. Therefore in order to prove the proposition it is sufficient to prove that the order of $\phi\left(\left[f_{1}\right]\right)$ is equal to the order of $\phi^{*}\left(\left[g_{1}\right]\right)$ where $\phi: H^{2}\left(G_{1}, U\left(L_{1}\right)\right)$ $\longrightarrow H^{2}\left(G_{1}, Z^{+}\right)$and $\phi^{*}: H^{2}\left(G_{1} G_{2}, U\left(L_{1} L_{2}\right)\right) \longrightarrow H^{2}\left(G_{1} G_{2}, Z^{+}\right)$are the maps used in the definition of the Brauer number.

Consider the following diagram.

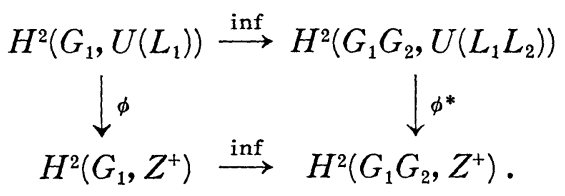

It is easy to verify that the above diagram is commutative. Therefore, in order to prove the proposition, it is sufficient to prove that the order of an element of $H^{2}\left(G_{1}, Z^{+}\right)$is preserved under the inflation map. So consider an element [h] of $H^{2}\left(G_{1}, Z^{+}\right)$, and let [ $\left.h^{*}\right]$ denote the image of [h] in $H^{2}\left(G_{1} G_{2}\right.$, $Z^{+}$). We proceed to show that the order $s$ of $[h]$ is equal to the order $t$ of $\left[h^{*}\right]$. The inequality $t \leq s$ is clear. To establish the opposite inequality we observe that since $t$ is the order of $\left[h^{*}\right]$ there exists a map $\Psi: G_{1} G_{2} \longrightarrow$ $Z^{+}$such that $\left(h^{*}(\alpha, \beta)\right)^{t}=\Psi(\alpha)+\Psi(\beta)-\Psi(\alpha \beta)$ for all elements $\alpha$ and $\beta$ of $G_{1} G_{2}$. The equalities $0=\left(h^{*}(1,1)\right)^{t}=\Psi(1)+\Psi(1)-\Psi(1)$ imply that $\Psi(1)=0$. 
Denote the Galois group of $L_{1} L_{2}$ over $L_{1}$ by $G$. We next observe that $\Psi(\alpha)=0$ whenever $\alpha$ is in the subgroup $G$ of $G_{1} G_{2}$. For since $h^{*}$ is the image of $h$ under inflation it follows that $0=(h(1,1))^{t}=\left(h^{*}(\alpha, \alpha)\right)^{t}=2 \Psi(\alpha)-$ $\Psi\left(\alpha^{2}\right)$ for $\alpha$ in $G$. Proceeding inductively one may show that $0=$ (ord $\alpha) \Psi(\alpha)-\Psi(\alpha$ ord $\alpha)$ where ord $\alpha$ denotes the order of the element $\alpha$ of $G$, and therefore $\Psi(\alpha)=0$ for every element $\alpha$ of $G$. Finally we observe that if $\bar{\alpha}=\bar{\beta}$ then $\Psi(\alpha)=\Psi(\beta)$, where $\bar{\alpha}$ denotes the image of $\alpha$ under the natural map of $G_{1} G_{2}$ onto $G_{1} G_{2} / G=G_{1}$. For, writing $\alpha$ in the form $\alpha=$ $\gamma \beta$ for some element $\gamma$ of $G$, and using the fact that $\Psi(\gamma)=0$, one may obtain the equalities $0=(h(\overline{1}, \bar{\beta}))^{t}=\left(h^{*}(\gamma, \beta)\right)^{t}=\Psi(\gamma)+\Psi(\beta)-\Psi(\gamma \beta)=\Psi(\beta)-\Psi(\alpha)$. Therefore $\Psi(\alpha)=\Psi(\beta)$. We may now consider the (well-defined) map $\theta$ : $G_{1} \longrightarrow Z^{+}$defined by $\theta(\bar{\alpha})=\Psi(\alpha)$. The fact that $(h(\bar{\alpha}, \bar{\beta}))^{t}=\theta(\bar{\alpha})+\theta(\bar{\beta})-\theta(\overline{\alpha \beta})$ implies that $[h]^{t}=[1]$. Therefore $s \leq t$, and this completes the proof.

We next define $T(k)$ to be the set of all elements of $V(k)$ whose Brauer numbers are relatively prime to the characteristic of $\bar{R}$. We adopt the convention that every number is relatively prime to zero, so that $T(k)=B(k)$ when $\bar{R}$ has characteristic zero.

The following lemma shall be useful in proving that $T(k)$ is a subgroup of $V(k)$.

Lemma 1.3. Let $\Sigma$ denote a central simple $k$-algebra such that $\widetilde{\Sigma}$ is in $V(k)$, and let $\Sigma^{0}$ denote the opposite ring of $\Sigma$. Then $\widetilde{\Sigma}$ and $\widetilde{\Sigma}^{0}$ have the same Brauer number.

Proof. Let $\Delta(f, L, G)$ be a representative of $\widetilde{\Sigma}$ with $L$ an unramified Galois extension of $k$. The $k$-algebra isomorphism $\Delta(f, L, G)^{0} \approx \Delta\left(f^{-1}, L, G\right)$ implies that $\widetilde{\Sigma}^{0}$ may be represented by $\Delta\left(f^{-1}, L, G\right)$. Consider the map $\phi: H^{2}(G, U(L)) \longrightarrow H^{2}\left(G, Z^{+}\right)$. Since $\phi([f])$ and $\phi\left(\left[f^{-1}\right]\right)$ have the same order it now follows that $\widetilde{\Sigma}$ and $\widetilde{\Sigma}^{0}$ have the same Brauer number.

Proposition 1.4. The set $T(k)$ is a subgroup of $V(k)$.

Proof. Let $\Sigma_{1}=\Delta\left(f_{1}, L_{1}, G_{1}\right)$ and $\Sigma_{2}=\Delta\left(f_{2}, L_{2}, G_{2}\right)$ be central simple $k$-algebras whose Brauer classes $\widetilde{\Sigma}_{1}$ and $\widetilde{\Sigma}_{2}$ are in $T(k)$, and let $n_{1}$ and $n_{2}$ denote the Brauer numbers of $\widetilde{\Sigma}_{1}$ and $\widetilde{\Sigma}_{2}$ respectively. Form the tensor product $\Sigma=\Sigma_{1} \otimes \Sigma_{2}^{0}$ and recall that the Brauer number of $\widetilde{\Sigma}_{2}^{0}$ is $n_{2}$ according to Lemma 1.3. To prove the proposition it suffices to show that the Brauer number of $\widetilde{\Sigma}$ is relatively prime to the characteristic of $\bar{R}$. 
For $i=1,2$ let $g_{i}$ denote the image of $f_{i}$ under the inflation map $Z^{2}\left(G_{i}, U\left(L_{i}\right)\right) \longrightarrow Z^{2}\left(G_{1} G_{2}, U\left(L_{1} L_{2}\right)\right)$ where $L_{1} L_{2}$ is the compositum of $L_{1}$ and $L_{2}$ and $G_{1} G_{2}$ is the Galois group of $L_{1} L_{2}$ over $k$, and observe that $\Sigma$ is equivalent to $\Delta\left(g_{1} g_{2}^{-1}, L_{1} L_{2}, G_{1} G_{2}\right)$. Consider the map $\phi: H^{2}\left(G_{1} G_{2}, U\left(L_{1} L_{2}\right)\right) \longrightarrow$ $H^{2}\left(G_{1} G_{2}, Z^{+}\right)$defined at the beginning of this section. Since $\left(\phi\left(\left[g_{1}\right]\right)\right)^{n_{1}}=[1]$ and $\left(\phi\left(\left[g_{2}^{-1}\right]\right)\right)^{n_{2}}=[1]$ it is clear that $\left(\phi\left(\left[g_{1} g_{2}^{-1}\right]\right)\right)^{n_{1} n_{2}}=[1]$ so that the Brauer number of $\widetilde{\Sigma}$ divides $n_{1} n_{2}$ and is hence relatively prime to the characteristic of $\bar{R}$.

REMARK 1.5. The subgroup $T(k)$ need not equal $V(k)$.

For consider the following example. Let $R=Z_{p}$ be the ring of $p$-adic integers, and $k=Q_{p}$ the quotient field of $R$. It is well known (see for example Prop. 3-2-12 of [9]) that there exists a (unique) unramified extension $L$ of $k$ with degree $p$. It is of the form $L=k(\zeta)$ where $\zeta$ denotes a primitive $\left(p^{p}-1\right)^{s t}$ root of unity. Furthermore, $L$ is a cyclic Galois extension of $k$ (see Remark 3-5-5 of [9]) and we denote the Galois group of $L$ over $k$ by $G$. Consider now the central simple $k$-algebra $\Sigma=\Delta(f, L, G)$ where $f$ is the element of $Z^{2}(G, U(L))$ which corresponds to $p \bmod N(U(L))$ under the canonical identification $H^{2}(G, U(L))=U(k) / N(U(L))$ which holds because $G$ is a cyclic group. It is easy to verify that the Brauer number of $\widetilde{\Sigma}$ is $p$ since $p$ is not a norm from $L$. We may conclude therefore that $T(k)$ is properly contained in $V(k)$.

We have thus defined the following chain of subgroups of the Brauer group

$$
B(k) \supset V(k) \supset T(k) \supset(1) \text {. }
$$

These groups shall be useful for studying equivalence classes of maximal orders over $R$.

We terminate Section 1 with some remarks concerning ramification. The ramification index of an hereditary order $\Lambda$ over a discrete rank one valuation ring $R$ can be defined according to Thm. 6. 1 of [5]. For let $\pi$ denote a prime element of $R$. Since the ideal $\pi \Lambda$ is an invertible $\Lambda$-ideal, there exists a positive integer $t$ such that $(\operatorname{rad} \Lambda)^{t}=\pi \Lambda$.

Definition. Let $\Lambda$ be an hereditary order over a discrete rank one valuation ring $R$, and let $\pi$ denote a prime element of $R$. The positive 
integer $t$ for which $(\operatorname{rad} \Lambda)^{t}=\pi \Lambda$ is called the ramification index of $\Lambda$ over $R$ and is denoted by $r(\Lambda / R)$.

Proposition 1.6. The ramification index of an hereditary order $\Lambda$ over a discrete rank one valuation ring $R$ depends only upon the equivalence class of $\Lambda$.

Proof. Let $\Omega$ denote an hereditary $R$-order which is equivalent to $A$. Then there exist finitely generated free $R$-modules $E_{1}$ and $E_{2}$ and an $R$ algebra isomorphism $\Lambda \otimes_{R} \operatorname{Hom}_{R}\left(E_{1}, E_{1}\right) \approx \Omega \otimes_{R} \operatorname{Hom}_{R}\left(E_{2}, E_{2}\right)$. Since $\operatorname{Hom}_{R}\left(E_{1}, E_{1}\right)$ is a central separable $R$-algebra it follows that $\operatorname{rad}\left(\Lambda \otimes_{R}\right.$ $\left.\operatorname{Hom}_{R}\left(E_{1}, E_{1}\right)\right)=(\operatorname{rad} \Lambda) \otimes_{R} \operatorname{Hom}_{R}\left(E_{1}, E_{1}\right)$ according to the proof of Prop. 8. 6 of [3]. Therefore $r\left(\Lambda \otimes_{R} \operatorname{Hom}_{R}\left(E_{1}, E_{1}\right) / R\right)=r(\Lambda / R)$, and similarly $r\left(\Omega \otimes_{R}\right.$ $\left.\operatorname{Hom}_{R}\left(E_{2}, E_{2}\right) / R\right)=r(\Omega / R)$. From the above isomorphism we may now conclude that $r(\Lambda / R)=r(\Omega / R)$.

Proposition 1.7. Let $k$ denote the quotient field of a complete discrete rank one valuation ring $R$, and $K$ a finite Galois extension of $k$ with Galois group $G$. If the integral closure $S$ of $R$ in $K$ is a tamely ramified extension of $R$, then the ramification index of a crossed product $\Delta(f, S, G)$ is equal to the ramification index of $S$ over $R$.

Proof. Since $S$ is a tamely ramified extension of $R$ it follows that the crossed product $\Delta=\Delta(f, S, G)$ is an hereditary $R$-order with radical $\Pi \Delta$ (see Prop. 1.3 of [10]), where $\Pi$ denotes a prime element of $S$. Hence $r(\Delta / R)=r(S / R)$.

2. The case of unequal characteristic. Let $R$ denote a complete discrete rank one valuation ring whose quotient field $k$ has characteristic zero, and whose residue class field $\bar{R}$ has characteristic $p \neq 0$. The purpose of this section is to prove that if $\widetilde{\Sigma}$ is an element of $V(k)$ whose Brauer number is equal to $p$, then a maximal order in $\Sigma$ is not equivalent to a crossed product over a tamely ramified extension of $R$.

Recall that an element $\widetilde{\Sigma}$ of $V(k)$ may be represented by a crossed product over an unramified Galois extension of $k$. Therefore, throughout this section $\Sigma$ shall denote a crossed product of the form $\Sigma=\Delta(f, L, G)$ where $L$ is an unramified extension of $k$, and it shall be assumed that the Brauer number of $\widetilde{\Sigma}$ is equal to the characteristic $p$ of $\bar{R}$.

In order to prove that a maximal order $\Gamma$ in such a central simple $k$ - 
algebra $\Sigma$ is not equivalent to a crossed product over a tamely ramified extension of $R$, we shall prove that the ramification index $r(\Gamma / R)$ of $\Gamma$ over $R$ is divisible by $p$, so that $\Gamma$ cannot be equivalent to a crossed product over a tamely ramified extension according to Prop. 1. 7 .

The method of proof is to reduce the problem to a study of crossed products by constructing a central simple $k$-algebra $\Sigma_{w}=\Delta\left(g, L_{w}, G_{w}\right)$ equivalent to $\Delta(f, L, G)$ and such that $g$ is in $Z^{2}\left(G_{w}, U\left(S_{w}\right)\right)$ where $S_{w}$, the integral closure of $R$ in $L_{w}$, is a wildly ramified extension of $R$. We shall then construct a maximal order $\Gamma_{w}$ in $\Sigma_{w}$ such that $\Gamma_{w}$ contains the crossed product $\Delta\left(g, S_{w}, G_{w}\right)$. Making use of this inclusion, we shall then prove that $r\left(\Gamma_{w} / R\right)=r\left(S_{w} / R\right)$.

In order to construct the desired crossed product $\Delta\left(g, L_{w}, G_{w}\right)$ we first construct the Galois extension $L_{w}$ of $k$. Let $L_{t}$ denote the extension of $L$ obtained by adjoining a primitive $p^{t h}$ root of unity. Observe that the extension $L_{t}$ of $L$ is tamely ramified since its degree is less than or equal to $p-1$. Let $S_{t}$ denote the integral closure of $R$ in $L_{t}$. According to Prop. 3-4-2 of [9] we may select prime elements $\pi$ and $\pi_{t}$ of $R$ and $S_{t}$ respectively in such a way that $\pi_{t}^{e}=\pi$ where $e$ denotes the ramification index of $S_{t}$ over $R$.

Now $L_{w}$ is defined to be the extension of $L_{t}$ obtained by adjoining a root $\Pi$ of the polynomial $F(X)=X^{p}-\pi_{t}$. Observe that $L_{w}$ is a Galois extension of $k$ since $L_{w}$ contains a primitive $p^{t h}$ root of unity; we denote the Galois group of $L_{w}$ over $k$ by $G_{w}$. The extension $L_{w}$ of $L_{t}$ is a wildly ramified inertial extension of degree $p$ and $\Pi$ is a prime element of the integral closure $S_{w}$ of $R$ in $L_{w}$. By virtue of Prop. 1. 2 we may as well assume that the integral closure $S$ of $R$ in $L$ is the inertia ring of $L_{w}$ over $k$.

Before constructing the 2-cocycle $g$ of $Z^{2}\left(G_{w}, U\left(S_{w}\right)\right)$ we summarize the ramification properties of the extension $S_{w}$ of $R$.

Lemma 2.1. Let $G_{i}$ denote the $i^{\text {th }}$ ramification group of $S_{w}$ over $R$. Then

i) $G_{1}$ is cyclic of order $p$

ii) $G_{0} / G_{1}$ is cyclic of order e relatively prime to $p$

iii) $G_{0}=G_{1} \times G_{0} / G_{1}$ (semi-direct product)

iv) $G_{i}=G_{1}$ and $G_{i+1}=(1)$ for $i=a /(p-1)$ where $a$ denotes the absolute ramification index of $L_{w}$. 
Proof. Assertion i) is clear from the definition of the extension $S_{w}$ of $S_{t}$. The second and third statements follow from Corollaries 1 and 4 respectively on p. 75 of [7], and the fourth statement follows from Ex. 4 p. 79 of [7].

The next lemma describes the action of the inertia group $G_{0}$ on the prime element $\Pi$ of $S_{w}$. According to Lemma 2.1 we may view $G_{0} / G_{1}$ as a subgroup of $G_{0}$.

Lemma 2.2. Let $\tau$ denote an element of $G_{1}$ and $\sigma$ an element of $G_{0} / G_{1}$. Then $\tau(\Pi)=\zeta_{\tau} \Pi$ for some $p^{t h}$ root of unity $\zeta_{\tau}$, and $\sigma(\Pi)=\xi_{\sigma} \Pi$ for some $e^{t h}$ root of unity $\xi_{\sigma}$, where $e$ denotes the order of $G_{0} / G_{1}$.

Proof. It is clear from the definition of $S_{w}$ that the conjugates of $\Pi$ relative to $S_{t}$ are of the form $\zeta^{2} \Pi$ for $1 \leq i \leq p$ where $\zeta$ denotes a primitive $p^{t h}$ root of unity, so that $\tau(\Pi)=\zeta_{\tau} \Pi$ for some $p^{t h}$ root of unity $\zeta_{\tau}$.

On the other hand we know that $S$ contains a primitive $e^{t h}$ root of unity $\xi$ according to Cor. 2-2-7 of [9]. Recall that the prime elements $\pi$ of $R$ and $\pi_{t}$ of $S_{t}$ were chosen so that $\pi_{t}^{e}=\pi$. Hence $X^{p e}-\pi$ is the minimal polynomial of $\Pi$ over $S$. The pe conjugates of $\Pi$ relative to $S$ are therefore given by $\zeta^{i} \xi^{j} \Pi$ for $1 \leq i \leq p$ and $1 \leq j \leq e$. If $\sigma$ is in $G_{0} / G_{1}$ then $\sigma^{e}(\Pi)=\Pi$ from which it follows that $\sigma(\Pi)=\xi_{\sigma}(\Pi)$ for some $e^{t h}$ root of unity $\xi_{\sigma}$.

Notation. Throughout the rest of this section $\tau$ shall denote a fixed generator of the cyclic group $G_{1}$, and $\zeta$ the primitive $p^{t h}$ root of unity defined by $\tau(\Pi)=\zeta \Pi$.

The group $G_{1}$ is a normal subgroup of $G_{w}$. For each element $\sigma$ of $G_{w}$ we may therefore consider the integer $n(\sigma)$ defined modulo $(p)$ by the equality $\sigma \tau \sigma^{-1}=\tau^{n(\sigma)}$. The next lemma presents properties of $n(\sigma)$ which shall be useful in this section.

Lemma 2.3. Let $n(\sigma)$ be defined as above. Then

i) $n(\sigma)=1$ if and only if $\sigma=1$ for $\sigma$ in $G_{0} / G_{1}$

ii) $\sigma(\zeta)=\zeta^{n(\sigma)}$ for each element $\sigma$ of $G_{w}$.

Proof. Consider an element $\sigma$ of $G_{0} / G_{1}$. By the definition of $n(\sigma)$ it follows that $n(\sigma)=1$ if and only if $\sigma \tau=\tau \sigma$ which holds if and only if $\sigma \tau(\Pi)=$ 
$\tau \sigma(\Pi)$ since $\tau$ is in $G_{1}$ and $S_{w}=S[\Pi]$. Let $\zeta_{\sigma}$ be the $e^{t h}$ root of unity satisfying $\sigma(\Pi)=\zeta_{\sigma} \Pi$. Then $\sigma \tau(\Pi)=\sigma(\zeta \Pi)=\zeta^{\sigma} \zeta_{\sigma} \Pi$ and $\tau \sigma(\Pi)=\tau\left(\zeta_{\sigma} \Pi\right)=$ $\zeta_{\sigma} \zeta \Pi$. Therefore $n(\sigma)=1$ if and only if $\zeta^{\sigma}=\zeta$, that is to say if and only if $\sigma=1$. This proves statement i).

Now according to Lemma 2.2 we know the conjugates of $\Pi$ relative to $k$. Therefore if $\sigma$ is an element of $G_{w}$ we have that $\sigma(\Pi)=u \Pi$ for some element $u$ of $U\left(S_{t}\right)$. Then $\sigma \tau \sigma^{-1}(\Pi)=\sigma \tau\left(\Pi / \sigma^{-1}(u)\right)=o\left(\zeta \Pi / \sigma^{-1}(u)\right)=$ $\zeta^{\sigma} \Pi$. But $\sigma \tau \sigma^{-1}(\Pi)=\tau^{n(\sigma)}(\Pi)=\zeta^{n(\sigma)} \Pi$, so that $\zeta^{\sigma}=\zeta^{n(\sigma)}$.

This completes the study of the extension $S_{w}$ of $R$, and we proceed now to construct the desired 2-cocycle $g$. The fact that the image of $[f]$ in $H^{2}\left(G, Z^{+}\right)$has order $p$ implies that there exists a map $\phi: G \longrightarrow U(L)$ such that the 2-cocycle $h$ of $Z^{2}(G, U(L))$ defined by $h(\sigma, \rho)=f^{p}(\sigma, \rho) \phi(\sigma \rho) / \phi(\sigma) \phi^{\sigma}(\rho)$ takes values in $U(S)$. Since $\phi(\sigma)$ is in $U(L)$ we may write $\phi(\sigma)$ in the form $\phi(\sigma)=\alpha_{\sigma} \pi^{\beta(\sigma)}$ where $\alpha_{\sigma}$ is in $U(S)$ and $\beta(\sigma)$ is an integer. Define the map $\phi_{w}: G_{w} \longrightarrow U\left(L_{w}\right)$ by $\phi_{w}(\sigma)=\Pi^{e \beta(\bar{\sigma})}$ where $e$ is the order of $G_{0} / G_{1}$ and $\bar{\sigma}$ denotes the image of $\sigma$ under the natural map of $G_{w}$ onto $G$. Let $f_{w}$ denote the image of $f$ under the inflation map $Z^{2}(G, U(L)) \longrightarrow Z^{2}\left(G_{w}, U\left(L_{w}\right)\right)$, and define the 2-cocycle $g$ of $Z^{2}\left(G_{w}, U\left(L_{w}\right)\right)$ by

$$
g(\sigma, \rho)=f_{w}(\sigma, \rho) \phi_{w}(\sigma \rho) / \phi_{w}(\sigma) \phi_{w}^{\sigma}(\rho) .
$$

Observe that $g$ is cohomologous to $f_{w}$ in $Z^{2}\left(G_{w}, U\left(L_{w}\right)\right)$ by definition, so that the central simple $k$-algebra $\Delta\left(g, L_{w}, G_{w}\right)$ is equivalent to $\Delta(f, L, G)$ (see for example Thm. 8. $5 E$ of [1]). The next three propositions present some useful properties of the 2-cocycle $g$.

Proposition 2.4. The element $g$ of $Z^{2}\left(G_{w}, U\left(L_{w}\right)\right)$ defined above is in the image of the natural map $Z^{2}\left(G_{w}, U\left(S_{t}\right)\right) \longrightarrow Z^{2}\left(G_{w}, U\left(L_{w}\right)\right)$.

Proof. We prove first that $g$ is in the image of the natural map $Z^{2}\left(G_{w}, U\left(S_{w}\right)\right) \longrightarrow Z^{2}\left(G_{w}, U\left(L_{w}\right)\right)$. In order to verify that $g$ takes values in $U\left(S_{w}\right)$ it clearly suffices to show that $g^{p}$ takes values in $U\left(S_{w}\right)$. From the definition of $g$ we obtain the equalities

$$
\begin{aligned}
g^{p}(\sigma, \rho) & =\left[f_{w}(\sigma, \rho) \phi_{w}(\sigma \rho) / \phi_{w}(\sigma) \phi_{w}^{\sigma}(\rho)\right]^{p} \\
& =\left[h(\bar{\sigma}, \bar{\rho}) \phi(\bar{\sigma}) \phi^{\bar{\sigma}}(\bar{\rho}) / \phi(\overline{\sigma \rho})\right]\left[\phi_{w}(\sigma \rho) / \phi_{w}(\sigma) \phi_{w}^{\sigma}(\rho)\right]^{p}
\end{aligned}
$$

where $\sigma$ and $\rho$ are elements of $G_{w}$, and $\bar{\sigma}$ denotes the image of $\sigma$ under 
the natural map of $G_{w}$ onto $G$. The definition of the map $\phi_{w}$ together with the equality $\Pi^{p e}=\pi$ implies that $\phi(\bar{\sigma}) /\left[\phi_{w}(\sigma)\right]^{p}=\alpha_{\bar{\sigma}}$ and $\phi^{\bar{\sigma}}(\bar{\rho}) /\left[\phi_{w}^{\sigma}(\rho)\right]^{p}$ $=\alpha_{\bar{\rho}}^{\bar{\sigma}}$. Therefore $g^{p}(\sigma, \rho)=h(\bar{\sigma}, \bar{\rho}) \boldsymbol{\alpha}_{\bar{\sigma}} \boldsymbol{\alpha}_{\bar{\rho}}^{\bar{\sigma}} / \boldsymbol{\alpha}_{\bar{\sigma} \bar{\rho}}$ from which it follows that $g^{p}$, and hence $g$, takes values in $U\left(S_{w}\right)$.

It remains to show that $g$ in fact takes values in $U\left(S_{t}\right)$. From the definitions of $g$ and $\phi_{w}$ we obtain the equality

$$
g(\sigma, \rho)=f_{w}(\sigma, \rho) \Pi^{e \beta(\overline{\sigma \rho})} / \Pi^{e \beta(\bar{\sigma})} \sigma\left(\Pi^{e \beta(\bar{\rho})}\right)
$$

for elements $\sigma$ and $\rho$ of $G_{w}$. According to Lemma 2.2 we may write $\sigma(\Pi)=\zeta_{\sigma} \xi_{\sigma} \Pi$ where $\zeta_{\sigma}$ and $\xi_{\sigma}$ are $p^{t h}$ and $e^{t h}$ roots of unity respectively. Since $f_{w}(\sigma, \rho)$ is in $U(L)$ we may write $f_{w}(\sigma, \rho)=\alpha_{\sigma, \rho} \pi^{\gamma(\sigma, \rho)}$ where $\alpha_{\sigma, \rho}$ is in $U(S)$ and $\gamma(\sigma, \rho)$ is in $Z$. Using the fact that $\Pi^{p e}=\pi$ one may now conclude that $\operatorname{per}(\sigma, \rho)+e \beta(\bar{\sigma} \bar{\rho})=e \beta(\bar{\sigma})+e \beta(\bar{\rho})$ since $g(\sigma, \rho)$ is in $U\left(S_{w}\right)$. Combining these observations we obtain that $g(\sigma, \rho)=\alpha_{\sigma, \rho} / \zeta_{\sigma}^{e \beta(\bar{\rho})}$. Therefore $g(\sigma, \rho)$ is in $U\left(S_{t}\right)$, since $\alpha_{\sigma, \rho}$ is in $U(S)$ and $\zeta_{\sigma}$ is in $U\left(S_{t}\right)$.

Proposition 2.5. The 2-cocycle g defined above has the following properties

i) $g(\sigma, \rho)=1$ for every $\sigma$ in $G_{w}$ and $\rho$ in $G_{0}$

ii) $g(\sigma, \rho)$ is a $p^{\text {th }}$ root of unity for every $\sigma$ in $G_{1}$ and $\rho$ in $G_{w}$

iii) $g(\sigma, \rho)=1$ for every $\sigma$ in $G_{0} / G_{1}$ and $\rho$ in $G_{w}$

iv) $\bar{g}$ is in the image of the inflation map $Z^{2}\left(G_{w} / G_{1}, U(\bar{S})\right) \longrightarrow Z^{2}\left(G_{w}, U(\bar{S})\right)$ where $\bar{g}$ denotes the image of $g$ under the natural map $Z^{2}\left(G_{w}, U\left(S_{w}\right)\right) \longrightarrow$ $Z^{2}\left(G_{w}, U(\bar{S})\right)$.

Proof. We first observe that $\beta(1)=0$ where $\beta: G \longrightarrow Z$ is the function used to define the 2-cocycle $g$. From the definition of $h$ we obtain the equalities $1=h(1,1)=f^{p}(1,1) \alpha_{1} \pi^{\beta(1)} /\left(\alpha_{1} \pi^{\beta(1)}\right)^{2}$ from which it follows that $1=$ $1 / \alpha_{1} \pi^{\beta(1)}$, so that $\beta(1)=0$.

Now let $\sigma$ denote an element of $G_{w}$ and $\rho$ an element of $G_{0}$, and observe that $f_{w}(\sigma, \rho)=f_{w}(\rho, \sigma)=1$ by the definition of $f_{w}$. Then $g(\sigma, \rho)=$ $f_{w}(\sigma, \rho) \phi_{w}(\sigma \rho) / \phi_{w}(\sigma) \phi_{w}^{\sigma}(\rho)=\Pi^{e \beta(\overline{\sigma \rho})} / \Pi^{e \beta(\bar{\sigma})} \sigma\left(\Pi^{e \beta(\bar{\rho})}\right)=1$ since $\bar{\rho}=\overline{1}$ and $\beta(\overline{1})=0$.

When $\sigma$ is in $G_{1}$ and $\rho$ is an element of $G_{w}$ we have the equalities $g(\sigma, \rho)=f_{w}(\sigma, \rho) \phi_{w}(\sigma \rho) / \phi_{w}(\sigma) \phi_{w}^{\sigma}(\rho)=\Pi^{e \beta(\overline{\sigma \rho})} / \Pi^{e \beta(\bar{\sigma})} \sigma\left(\Pi^{e \beta(\bar{\rho})}\right)=\Pi^{e \beta(\bar{\rho})} / \sigma\left(\Pi^{e \beta(\bar{\rho})}\right)$. Therefore $g(\sigma, \rho)$ is a $p^{t h}$ root of unity since $\sigma\left(\Pi^{e \beta(\bar{\rho})}\right)=[\sigma(\Pi)]^{e \beta(\bar{\rho})}=\left(\zeta_{\sigma} \Pi\right)^{e \beta(\bar{\rho})}$ for some $p^{t h}$ root of unity $\zeta_{\sigma}$ since $\sigma$ is in $G_{1}$. 
Next let $\sigma$ denote an element of $G_{0} / G_{1}$ and $\rho$ any element of $G_{w}$. Then $g(\sigma, \rho)=\Pi^{e \beta(\tilde{\sigma})} / \sigma\left(\Pi^{e \beta(\bar{\rho})}\right)$. Since $\sigma(\Pi)=\xi_{\sigma} \Pi$ for some $e^{t h}$ root of unity $\xi_{\sigma}$ according to Lemma 2.2 we may conclude that $g(\sigma, \rho)=1$.

Finally, in order to prove assertion iv) it suffices to observe that $\bar{g}(\sigma, \rho)$ $=\bar{g}(\rho, \sigma)=\overline{1}$ for $\sigma$ in $G_{1}$ and $\rho$ in $G_{w}$ according to parts i) and ii) of this proposition. One can then verify that the map $q: G_{w} / G_{1} \times G_{w} / G_{1} \longrightarrow U(\bar{S})$ defined by $q(\bar{\sigma}, \bar{\rho})=g(\sigma, \rho)$ is an element of $Z^{2}\left(G_{w} / G_{1}, U(\bar{S})\right)$ in the preimage of $g$.

Proposition 2.6. There exists an element $\sigma$ in $G_{w}$ such that $g\left(\tau^{n(\sigma)}, \sigma\right) \neq 1$.

Proof. We first prove by contradiction that there exists an element $\sigma$ of $G_{w}$ for which $g(\tau, \sigma) \neq 1$. For suppose that $g(\tau, \sigma)=1$ for each element $\sigma$ of $G_{w}$. From the proof of part ii) of Prop. 2. 5 we know that $g(\tau, \sigma)=\zeta^{e \beta(\bar{\sigma})}$ where $\zeta$ is the primitive $p^{t h}$ root of unity defined by $\tau(\Pi)=\zeta \Pi$. Since $e$ is relatively prime to $p$, the assumption that $g(\tau, \sigma)=1$ implies that $\beta(\tilde{\sigma})$ is divisible by $p$. For each element $\sigma$ of $G_{w}$ we may define an integer $\gamma(\bar{\sigma})$ by $\beta(\bar{\sigma})=\operatorname{pr}(\bar{\sigma})$ where $\bar{\sigma}$ denotes the image of $\sigma$ under the natural map of $G_{w}$ onto $G_{w} / G_{0}=G$. We proceed to prove that $[f]$ is in the image of the natural map $H^{2}(G, U(S)) \longrightarrow H^{2}(G, U(L))$ and thus contradict the assumption on the Brauer number of $\Sigma$ where $\Sigma=\Delta(f, L, G)$. Return once again to the notation used in the definition of $g$. We may now express the 2cocycle $h$ of $Z^{2}(G, U(S))$ in the form $h(\sigma, \rho)=f^{p}(\sigma, \rho) \alpha_{\sigma \rho} \pi^{p \gamma(\sigma \rho)} / \alpha_{\sigma} \pi^{p \gamma(\sigma)} \sigma\left(\alpha_{\rho} \pi^{p \gamma(\rho)}\right)$. Define the map $\Psi: G \longrightarrow U(L)$ by $\Psi(\sigma)=\pi^{r(\sigma)}$. Then the 2-cocycle $q$ of $Z^{2}(G, U(L))$ defined by $q(\sigma, \rho)=f(\sigma, \rho) \Psi(\sigma \rho) / \Psi(\sigma) \Psi^{\sigma}(\rho)$ is cohomologous to $f$ and takes values in $U(S)$, so that $[f]$ is in the image of the natural map $H^{2}(G, U(S)) \longrightarrow H^{2}(G, U(L))$. From this contradiction we conclude that there exists an element $\sigma$ of $G_{w}$ for which the $p^{t h}$ root of unity $g(\tau, \sigma)$ is not equal to 1 .

It remains to prove that $g\left(\tau^{n(\sigma)}, \sigma\right) \neq 1$ for this element $\sigma$. We first prove inductively that $g\left(\tau^{i}, \sigma\right)=(g(\tau, \sigma))^{i}$. The assertion is trivial for $i=1$. So we must prove that $g\left(\tau^{i+1}, \sigma\right)=(g(\tau, \sigma))^{i+1}$ under the assumption that $g\left(\tau^{i}, \sigma\right)=(g(\tau, \sigma))^{i}$. From the associativity property of $g$ we obtain the equality $g\left(\tau^{i+1}, \sigma\right) g\left(\tau^{i}, \tau\right)=g\left(\tau^{i}, \tau \sigma\right) g^{\tau^{i}}(\tau, \sigma)$, so that $g\left(\tau^{i+1}, \sigma\right)=g\left(\tau^{i}, \tau \sigma\right) g(\tau, \sigma)$ since $g\left(\tau^{i}, \tau\right)=1$ and $g(\tau, \sigma)$ is in $U\left(S_{t}\right)$. Now $g\left(\tau^{i}, \tau \sigma\right)=g\left(\tau^{i}, \sigma \tau^{n\left(\sigma^{-1}\right)}\right)$ so that we obtain once again by associativity the equality $g\left(\tau^{i}, \tau \sigma\right) g^{\tau^{i}}\left(\sigma, \tau^{n\left(\sigma^{-1}\right)}\right)=$ 
$g\left(\tau^{i} \sigma, \tau^{n\left(\sigma^{-1}\right)}\right) g\left(\tau^{i}, \sigma\right)$ from which it follows that $g\left(\tau^{i}, \tau \sigma\right)=g\left(\tau^{i}, \sigma\right)$ according to part i) of Prop. 2. 5. Combining these results we conclude that $g\left(\tau^{i+1}, \sigma\right)=$ $g\left(\tau^{i}, \sigma\right) g(\tau, \sigma)$. The induction hypothesis now implies that $g\left(\tau^{i+1}, \sigma\right)=(g(\tau, \sigma))^{i+1}$. Therefore $g\left(\tau^{n(\sigma)}, \sigma\right)=(g(\tau, \sigma))^{n(\sigma)}$. Since $g(\tau, \sigma)$ is a $p^{t h}$ root of unity different from 1 , and $n(\sigma)$ is relatively prime to $p$, we conclude at last that $g\left(\tau^{n(\sigma)}, \sigma\right) \neq 1$.

For convenience of notation we denote the crossed product $\Delta\left(g, S_{w}, G_{w}\right)$ by $\Delta_{w}$ and $\Delta\left(g, L_{w}, G_{w}\right)$ by $\Sigma_{w}$. Observe that $\Delta_{w}$ is an $R$-order in $\Sigma_{w}$.

We next construct an order $\Gamma_{w}$ in $\Sigma_{w}$ containing the crossed product $\Delta_{w}$. Let $\theta$ denote the element of $\Sigma_{w}$ defined by $\theta=\frac{1}{1-\zeta}\left(u_{\tau}-1\right)$. Then $\Gamma_{w}$ is defined to be the ring obtained by adjoining the element $\theta$ to $\Delta_{w}$; i.e. $\Gamma_{w}=\Delta_{w}[\theta]$. Most of this section is devoted to proving that $\Gamma_{w}$ is a maximal order and to the computation of its unique maximal two-sided ideal.

We must first verify that the ring $\Gamma_{w}$ defined above is in fact an order in $\Sigma_{w}$. Since $\Gamma_{w}$ contains $\Delta_{w}$, it is clear that $\Gamma_{w}$ spans $\Sigma_{w}$ over $k$. To prove that $\Gamma_{w}$ is a finitely generated $R$-module, we show next that $\theta$ satisfies a polynomial equation over the subring $\Delta\left(1, S_{w}, G_{1}\right)$ of $\Delta_{w}$. Observe that $\theta$ is an element of the subring $\Delta\left(1, L_{w}, G_{1}\right)$ of $\Sigma_{w}$.

Lemma 2.7. Let $Z_{p}$ denote the ring of $p$-adic integers, and $\zeta$ a primitive $p^{\text {th }}$ root of unity. Let $v$ denote the element of $Z_{p}$ defined by the equality $(1-\zeta)^{p-1}=$ $v p$. Then $v \equiv-1 \bmod (1-\zeta)$.

Proof. Since $Z_{p}[\zeta]$ is a tamely ramified inertial extension of $Z_{p}$ of degree $p-1$ and with prime element $1-\zeta$, it is clear that $(1-\zeta)^{p-1}=v p$ for some unit $v$ of $Z_{p}[\zeta]$. It is a well known fact (see p. 258 of [9]) that $p=$ $(1-\zeta)\left(1-\zeta^{2}\right) \cdots\left(1-\zeta^{p-1}\right)$. The equality $1-\zeta^{i}=(1-\zeta)\left(1+\zeta+\cdots+\zeta^{i-1}\right)$ implies that $\zeta^{i} \equiv 1 \bmod (1-\zeta)$ for every integer $i$, so that $1+\zeta+\cdots+\zeta^{i}$ $\equiv i+1 \bmod (1-\zeta)$. Factoring the right hand side of the above expression for $p$ we obtain the equality $p=(1-\zeta)^{p-1}(1+\zeta)\left(1+\zeta+\zeta^{2}\right) \cdots(1+\zeta$ $\left.+\cdots+\zeta^{p-2}\right)$. But according to the above, $(1+\zeta)\left(1+\zeta+\zeta^{2}\right) \cdots(1+\zeta$ $\left.+\cdots+\zeta^{p-2}\right) \equiv(p-1) ! \bmod (1-\zeta)$. By Wilson's theorem (p. 118 of [8]) we know that $(p-1) ! \equiv-1 \bmod (p) . \quad$ Therefore $v \equiv-1 \bmod (1-\zeta)$.

Lemma 2.8. Let $C_{i}$ be the integer defined for $1 \leq i \leq \frac{p-1}{2}$ by $C_{i}=$ 
$(-1)^{i}\left[\frac{(p-1)(p-2) \cdot \cdots(p-i+1)}{i !}\right](p-2 i) . \quad$ Then $C_{1}+C_{2}+\cdots+C_{\frac{p-1}{2}}$ $\equiv-1 \bmod (p)$.

Proof. Since $C_{i} \equiv(-2)(-1)^{i}(p-1) \cdots(p-i+1) /(i-1) ! \bmod (p)$, it suffices to observe that $(p-1)(p-2) \cdots(p-(i-1)) \equiv(-1)^{i-1}(i-1) ! \bmod$ $(p)$ in order to establish the fact that $C_{i} \equiv 2 \bmod (p)$ for $1 \leq i \leq(p-1) / 2$. Therefore $C_{1}+\cdots+C_{(p-1) / 2} \equiv-1 \bmod (p)$ since there are $(p-1) / 2$ summands.

Lemma 2.9. Let $\Delta_{1}=\Delta\left(1, S_{w}, G_{1}\right)$ and consider the left $\Delta_{1}$-submodule $\Delta_{1}\left(u_{\tau}-1\right.$, $\left.\left(u_{\tau}-1\right) \theta\right)$ of $\Delta\left(1, L_{w}, G_{1}\right)$ generated by the elements $u_{\tau}-1$ and $\left(u_{\tau}-1\right) \theta$. The element $\theta$ has the property that $\theta^{p}-\theta$ is in $\Delta_{1}\left(u_{\tau}-1,\left(u_{\tau}-1\right) \theta\right)$.

Proof. We consider first the case of an odd prime $p$. Observe that $1-\zeta$ is in the center of $\Delta\left(1, L_{w}, G_{1}\right)$. By expanding $\left(u_{\tau}-1\right)^{p}$ according to the binomial theorem and combining terms with the same binomial coefficient one may obtain the equality

$$
\theta^{p}=\frac{p}{(1-\zeta)^{p}-1} \sum_{i}(-1)^{i}\left[\frac{(p-1) \cdot \cdots(p-i+1)}{i !}\right]\left[\frac{1}{1-\zeta}\left(u_{\tau}^{p-2 i}-1\right) u_{\tau}^{i}\right]
$$

for $1 \leq i \leq(p-1) / 2$. For convenience of notation let $A_{i}=(-1)^{i}(p-1) \cdot \cdots$ $(p-i+1) / i$ !. By writing $u_{\tau}^{p-2 i}-1$ in the form $u_{\tau}^{p-2 i}-1=\left(u_{\tau}^{p-2 i-1}+\cdots\right.$ $+1)\left(u_{\tau}-1\right)$ we have that

$$
\theta^{p}=\frac{p}{(1-\zeta)^{p-1}}\left[\sum A_{i}\left(u_{\tau}^{p-i-1}+\cdots+u_{\tau}^{i}\right)\right] \theta
$$

We next observe that $\left(u_{\tau}^{p-i-1}+\cdots+u_{\tau}{ }^{i}\right)-(p-2 i)$ is in $\Delta_{1}\left(u_{\tau}-1\right)$, since there are precisely $p-2 i$ summands in the expression $u_{\tau}^{p-i-1}+\cdots$ $+u_{\tau}{ }^{i}$. This now implies that $\theta^{p}-\frac{p}{(1-\zeta)^{p-1}}\left[\sum A_{i}(p-2 i)\right] \theta$ is in $\Delta_{1}\left(\left(u_{\tau}-1\right) \theta\right)$. Since $p /(1-\zeta)^{p_{-1}} \equiv-1 \bmod (1-\zeta)$ (see Lemma 2.7) and $\sum A_{i}(p-2 i) \equiv-1 \bmod (p)$ according to Lemma 2.8 we conclude at last that $\theta^{p}-\theta$ is in $\Delta_{1}\left(u_{\tau}-1,\left(u_{\tau}-1\right) \theta\right)$.

In the case $p=2$, one can verify by an easy computation that $\theta^{2}-\theta=$ $-2 \theta=-\left(u_{\tau}-1\right)$ and this completes the proof.

Corollary 2.10. The element $\theta$ of the ring $\Gamma_{w}$ has the property that $\theta^{p}-\theta$ $\equiv 0 \bmod (1-\zeta) \Gamma_{w}$. 
Proof. The proof is immediate from the lemma since the element $u_{\tau}$ 1 is in $(1-\zeta) \Gamma_{w}$.

Proposition 2.11. The ring $\Gamma_{w}$ is generated as both a left and a right $\Delta\left(g, S_{w}, G_{w}\right)$-module by $\left\{1, \theta, \cdots, \theta^{p-1}\right\}$.

Proof. We prove first that $\Gamma_{w}$ is generated as a right $\Delta_{w}$-module by powers of $\theta$. The inclusion $\Delta_{w}(\theta) \subset(1, \theta) \Delta_{w}$ may be obtained by showing that $\left(\alpha_{\rho} u_{\rho}\right) \theta$ is contained in $(1, \theta) \Delta_{w}$ for every element $\rho$ of $G_{w}$ and $\alpha_{\rho}$ of $S_{w}$. Using the fact that $g\left(\tau^{n(\rho)}, \rho\right)=\eta$ is a $p^{t h}$ root of unity (see Prop. 2. 5) together with the fact that $\left(1-\zeta^{\rho}\right)=v(1-\zeta)$ for some element $v$ of $U\left(S_{t}\right)$ one may obtain the equality

$$
\left(\alpha_{\rho} u_{\rho}\right) \theta=\frac{1}{1-\zeta}\left[u_{\tau}^{n(\rho)} \tau^{-n(\rho)}\left(\alpha_{\rho} / \eta v\right)-\alpha_{\rho} / v\right] u_{\rho} .
$$

Part iv) of Lemma 2.1 implies that $\tau^{-n(\rho)}\left(\alpha_{\rho} / \eta v\right) \equiv \alpha_{\rho} / \eta v \bmod (\Pi(1-\zeta))$. Since $\eta \equiv 1 \bmod (\Pi(1-\zeta))$ we may write $\tau^{-n(\rho)}\left(\alpha_{\rho} / \eta v\right)=\alpha_{\rho} / v+s \Pi(1-\zeta)$ for some element $s$ of $S_{w}$. Writing $u_{\tau}^{n(\rho)}-1$ in the form $u_{\tau}^{n(\rho)}-1=$ $\left(u_{\tau}-1\right)\left(u_{\tau}^{n(\rho)-1}+\cdots+1\right)$ we next obtain that

$$
\left(\alpha_{\rho} u_{\rho}\right) \theta=\theta\left(u_{\tau}^{n(\rho)-1}+\cdots+1\right)\left(\alpha_{\rho} / v\right)+\left(u_{\tau}^{n(\rho)}-1\right) s \Pi
$$

from which it may be seen at once that $\Delta_{w}(\theta)$ is contained in $(1, \theta) \Delta_{w}$.

It now follows inductively that $\Delta_{w}\left(\theta^{i}\right)$ is contained in $\left(\theta^{i-1}, \theta^{i}\right) \Delta_{w}$ for every positive integer $i$. Since $\theta$ satisfies an equation of degree $p$ over $\Delta\left(1, S_{w}, G_{1}\right)$ (see Lemma 2.9) we conclude that $\Gamma_{w}$ is generated as a right $\Delta_{w}$-module by $\left\{1, \theta, \ldots, \theta^{p-1}\right\}$.

By a similar computation one can show that $\Gamma_{w}$ is generated as a left $\Delta_{w}$-module by $\left\{1, \theta, \ldots, \theta^{p_{-1}}\right\}$.

Proposition 2.12. The ring $\Gamma_{w}$ is an $R$-order in the central simple $k$ algebra $\Delta\left(g, L_{w}, G_{w}\right)$.

Proof. To prove that $\Gamma_{w}$ is an order in $\Sigma_{w}=\Delta\left(g, L_{w}, G_{w}\right)$ we must show that $\Gamma_{w}$ is a finitely generated $R$-module such that $k \Gamma_{w}=\Sigma_{w}$. Since $\Delta_{w}=$ $\Delta\left(g, S_{w}, G_{w}\right)$ is an order in $\Sigma_{w}$ and $\Gamma_{w}$ contains $\Delta_{w}$, it follows that $k \Gamma_{w}=$ $\Sigma_{w}$. And, $\Gamma_{w}$ is a finitely generated $R$-module since $\Gamma_{w}$ is a finitely generated $\Delta_{w}$-module and $\Delta_{w}$ is a finitely generated $R$-module. 
The object now is to prove that the radical of $\Gamma_{w}$ is generated by the prime element $\Pi$ of $S_{w}$. The following general observation concerning orders shall be useful, (see Lemma 1. 7 of [13]) .

Lemma 2.13. Let $R$ denote a discrete rank one valuation ring with quotient field $k$, and let $\Lambda_{1}$ and $\Lambda_{2}$ be orders over $R$ in the same central simple k-algebra. If $\left(\mathrm{rad} \Lambda_{2}\right) \cap \Lambda_{1}$ is a two-sided ideal of $\Lambda_{1}$, then ( $\left.\mathrm{rad} \Lambda_{2}\right) \cap \Lambda_{1}$ is contained in rad $\Lambda_{1}$. In particular if $\Lambda_{1}$ is contained in $\Lambda_{2}$, then $\left(\mathrm{rad} \Lambda_{2}\right) \cap \Lambda_{1}$ is contained in rad $\Lambda_{1}$

Proof. Let $\pi$ denote the prime element of $R$. The fact that $\pi \Lambda_{2}=\Lambda_{2} \pi$ together with the fact that $\Lambda_{2}$ is a finitely generated left $R$-module implies that $\pi$ is contained in $\operatorname{rad} \Lambda_{2}$ (see Lemma 1.4 of [12]). And for similar reasons $\pi$ is contained in $\operatorname{rad} \Lambda_{1}$.

The residue class ring $\Lambda_{2} / \pi \Lambda_{2}$ is an Artin ring, so that its radical is nilpotent. Let $x$ be a positive integer for which $\left(\operatorname{rad} \Lambda_{2} / \pi \Lambda_{2}\right)^{x}=(0)$ and observe that $\left(\operatorname{rad} \Lambda_{2}\right)^{x}$ is contained in $\pi \Lambda_{2}$. Since $\Lambda_{1}$ and $\Lambda_{2}$ are orders in the same central simple $k$-algebra there exists a positive integer $y$ such that $\pi^{y} \Lambda_{2}$ is contained in $\Lambda_{1}$ (see p. 2 of [4]). Combining these observations we now obtain that $\left[\left(\operatorname{rad} \Lambda_{2}\right) \cap \Lambda_{1}\right]^{x(y+1)}$ is contained in $\pi \Lambda_{1}$. It now follows from the assumption on $\left(\operatorname{rad} \Lambda_{2}\right) \cap \Lambda_{1}$ that its image under the natural map of $\Lambda_{1}$ onto $\Lambda_{1} / \pi \Lambda_{1}$ is a nilpotent two-sided ideal. Using the fact that $\Lambda_{1} / \pi \Lambda_{1}$ is an Artin ring we may now conclude that $\left(\operatorname{rad} \Lambda_{2}\right) \cap \Lambda_{1}$ is contained in $\operatorname{rad} \Lambda_{1}$.

If $\Lambda_{1}$ is contained in $\Lambda_{2}$, then $\left(\operatorname{rad} \Lambda_{2}\right) \cap \Lambda_{1}$ is a two-sided ideal of $\Lambda_{1}$ and is therefore contained in $\operatorname{rad} \Lambda_{1}$ according to the above.

Lemma 2.14. Let $\Pi$ denote the prime element of $S_{w}$. Then

i) $\Pi \Gamma_{w}=\Gamma_{w} \Pi$

ii) $\Pi$ is contained in rad $\Gamma_{w}$

iii) $\operatorname{rad} \Delta_{w}=\left(\Pi, u_{\tau}-1\right) \Delta_{w}=\Delta_{w}\left(\Pi, u_{\tau}-1\right)$

iv) $\Gamma_{w} \Pi \cap \Delta_{w}=\operatorname{rad} \Delta_{w}$.

Proof. Since $\Delta_{w} \Pi=\Pi \Delta_{w}$ because $\Delta_{w}$ is a crossed product over $S_{w}$, it suffices to show that $\theta^{i} \Pi$ is in $\Pi \Gamma_{w}$ for $1 \leq i \leq p-1$ in order to obtain the inclusion $\Gamma_{w} \Pi \subset \Pi \Gamma_{w}$. Now $\theta \Pi=\Pi \theta+\frac{\Pi}{1-\zeta}\left[\frac{\tau(\Pi)}{\Pi}-1\right] u_{\tau}$ so that $\theta \Pi$ 
is in $\Pi \Gamma_{w}$ because $\frac{1}{1-\zeta}\left[\frac{\tau(\Pi)}{\Pi}-1\right]$ is in $S_{t}$. It follows inductively that $\theta^{i} \Pi$ is in $\Pi \Gamma_{w}$ for $1 \leq i \leq p-1$ so that $\Gamma_{w} \Pi$ is contained in $\Pi \Gamma_{w}$. The opposite inclusion may be obtained by a similar computation, and therefore $\Gamma_{w} \Pi=\Pi \Gamma_{w}$.

The fact that $\Pi \Gamma_{w}=\Gamma_{w} \Pi$ implies that $\Pi$ is in $\operatorname{rad} \Gamma_{w}$ according to Lemma 1. 4 of [12].

In order to prove iii) we first observe that the radical of the subring $\Delta_{1}=\Delta\left(1, S_{w}, G_{1}\right)$ of $\Delta_{w}$ is generated as a right ideal by $\Pi$ and $u_{\tau}-1$ where $\tau$ denotes as usual a generator of $G_{1}$. For, the $\bar{S}$-algebra isomorphism $\Delta\left(1, \bar{S}, G_{1}\right) \approx \bar{S}[X] /\left(X^{p}-1\right)$ induced by defining $u_{\tau} \longrightarrow X$ implies that the radical of the commutative ring $\Delta\left(1, \bar{S}, G_{1}\right)$ is generated by $u_{\tau}-1$. The natural isomorphism $\Delta_{1} / \Pi \Delta_{1} \approx \Delta\left(1, \bar{S}, G_{1}\right)$ together with the fact that $\Pi$ is in $\operatorname{rad} \Delta_{1}$ implies that $\operatorname{rad} \Delta_{1}=\left(\Pi, u_{\tau}-1\right) \Delta_{1}$. Now Props. 3. 1 and 3. 4 of [12] together imply that $\operatorname{rad} \Delta_{W}=\left(\operatorname{rad} \Delta_{1}\right) \Delta_{w}$. Combining the above observations we conclude that $\operatorname{rad} \Delta_{w}=\left(\Pi, u_{\tau}-1\right) \Delta_{w}$.

Now we may prove iv). The equality $u_{\tau}-1=\theta(1-\zeta)$ implies that $u_{\tau}-1$ is in $\Gamma_{w} \Pi \cap \Delta_{w}$ since $1-\zeta$ is in $S_{w}$. Since $\operatorname{rad} \Delta_{w}=\left(\Pi, u_{\tau}-1\right) \Delta_{w}$ according to part iii), we may conclude that $\operatorname{rad} \Delta_{w}$ is contained in $\Gamma_{w} \Pi \cap \Delta_{w}$. On the other hand, the intersection $\Gamma_{w} \Pi \cap \Delta_{w}$ is contained in rad $\Delta_{w}$ by Lemma 2.13. This completes the proof of statement iv).

Since $\Gamma_{w} \Pi$ is a two-sided ideal of $\Gamma_{w}$ we may form the residue class ring $\Gamma_{w} / \Gamma_{w} \Pi$, which shall henceforth be denoted by $\bar{\Gamma}_{w}$. According to Prop. 2.5 we may consider an element of $Z^{2}\left(G_{w} / G_{1}, U(\bar{S})\right)$ in the preimage of $\bar{g}$ under the inflation map $Z^{2}\left(G_{w} / G_{1}, U(\bar{S})\right) \longrightarrow Z^{2}\left(G_{w}, U(\bar{S})\right)$ which for convenience of notation shall also be denoted by $\bar{g}$. The following isomorphism shall be useful in establishing the semi-simplicity of $\bar{\Gamma}_{w}$.

Lemma 2.15. The residue class ring $\bar{\Gamma}_{w}$ is $\bar{R}$-algebra isomorphic to $\Delta(\bar{g}, \bar{S}$, $\left.G_{w} / G_{1}\right)[\bar{\theta}]$ in a natural way, where $\bar{\theta}$ denotes the residue class of $\theta$ modulo $\Gamma_{w} \Pi$.

Proof. Using the fact that $\bar{g}$ is in the image of the inflation map $Z^{2}\left(G_{w} / G_{1}, U(\bar{S})\right) \longrightarrow Z^{2}\left(G_{w}, U(\bar{S})\right)$ we may observe that the crossed product $\Delta\left(\bar{g}, \bar{S}, G_{w} / G_{1}\right)$ is isomorphic to $\Delta_{w} /\left(\Pi, u_{\tau}-1\right) \Delta_{w}$ in a natural way. Parts iii) and iv) of Lemma 2.14 imply that $\Gamma_{w} \Pi \cap \Delta_{w}=\left(\Pi, u_{\tau}-1\right) \Delta_{w}$, so that 
there is a natural injection of $\Delta_{w} /\left(\Pi, u_{\tau}-1\right) \Delta_{w}$ into $\bar{\Gamma}_{w} \cdot \quad$ By identifying $\Delta\left(\bar{g}, \bar{S}, G_{w} / G_{1}\right)$ with its image under the maps

$$
\Delta\left(\bar{g}, \bar{S}, G_{w} / G_{1}\right) \longrightarrow \Delta_{w} /\left(\Pi, u_{\tau}-1\right) \Delta_{w} \longrightarrow \bar{\Gamma}_{w}
$$

we may conclude that $\bar{\Gamma}_{w}$ is $R$-algebra isomorphic to $\Delta\left(\bar{g}, \bar{S}, G_{w} / G_{1}\right)[\bar{\theta}]$ since $\Gamma_{w}$ is generated as a left $\Delta_{w}$-module by $\left\{1, \theta, \ldots, \theta^{p-1}\right\}$.

Lemma 2.16 A. The intersection $\left(\Pi \Gamma_{w}\right) \cap \Delta\left(g, S_{w}, G_{0}\right)[\theta]$ is contained in $\Pi \Delta\left(g, S_{w}, G_{0}\right)[\theta]$.

Proof. Consider an element $\delta$ of $\left(\Pi \Gamma_{w}\right) \cap \Delta\left(g, S_{w}, G_{0}\right)[\theta]$. Since $\delta$ is in $\Pi \Gamma_{w}$ we may write $\delta$ in the form $\delta=\Pi \sum \delta_{i} \theta^{i}$ with the $\delta_{i}$ in $\Delta\left(g, S_{w}, G_{w}\right)$ according to Prop. 2.11.

We now use some properties of crossed products to show that each $\delta_{i}$ is in $\Delta\left(g, L_{w}, G_{0}\right)$. Since $\Delta\left(g, S_{w}, G_{0}\right)[\theta]$ is contained in $\Delta\left(g, L_{w}, G_{0}\right)$ it follows that $\sum \delta_{i} \theta^{i}$ is in $\Delta\left(g, L_{w}, G_{0}\right)$. Consider a disjoint (left) coset decomposition $G_{w}=\cup \omega_{j} G_{0}$ of $G_{w}$ with respect to the subgroup $G_{0}$, with $\omega_{1}=1$. According to Lemma 2.5 of [12], $\Delta\left(g, L_{w}, G_{w}\right)$ is a free right $\Delta\left(g, L_{w}, G_{o}\right)$-module with free basis $\left\{u_{\omega_{j}}\right\}$. Since each $\delta_{i}$ is in $\Delta\left(g, S_{w}, G_{w}\right)$ we may therefore write $\delta_{i}$ uniquely in the form $\delta_{i}=\sum u_{\omega_{j}} \delta_{j}^{(i)}$ where the $\delta_{j}^{(i)}$ are elements of $\Delta\left(g, L_{w}, G_{0}\right)$. The equality $\sum \delta_{i} \theta^{i} \equiv \sum_{j} u_{\omega_{j}}\left(\sum \delta_{j}^{(i)} \theta^{i}\right)$ now implies that $j=1$ because $\sum \delta_{i} \theta^{i}$ is in $\Delta\left(g, L_{w}, G_{0}\right)$. Therefore $\delta_{i}=\delta_{1}^{(i)}$ for each $i$, and so each $\delta_{i}$ is in $\Delta\left(g, L_{w}, G_{0}\right)$.

Using the fact that $\Delta\left(g, L_{w}, G_{w}\right)$ is a free (left) $L_{w}$-module with free basis $\left\{u_{\sigma}\right\}$ for all $\sigma$ in $G_{w}$, it is easy to see that the intersection $\Delta\left(g, S_{w}, G_{w}\right) \cap$ $\Delta\left(g, L_{w}, G_{0}\right)$ is contained in $\Delta\left(g, S_{w}, G_{0}\right)$. Therefore each $\delta_{i}$ is in $\Delta\left(g, S_{w}, G_{0}\right)$ and hence $\delta$ is in $\Pi \Delta\left(g, S_{w}, G_{0}\right)[\theta]$.

Lemma 2.16 B. The subring $\Delta\left(1, \bar{S}, G_{0} / G_{1}\right)[\bar{\theta}]$ of $\bar{\Gamma}_{w}$ is a commutative semisimple ring.

Proof. We prove first that the ring $\Delta\left(1, \bar{S}, G_{0} / G_{1}\right)[\bar{\theta}]$ is commutative. Now the crossed product $\Delta\left(1, \bar{S}, G_{0} / G_{1}\right)$ is commutative because $G_{0} / G_{1}$ is a cyclic group with trivial action on $\bar{S}$. Let $\rho$ denote a generator of $G_{0} / G_{1}$. Since $\bar{\theta}$ commutes with the elements of $\bar{S}$ it suffices to show that $u_{\rho}$ commutes with $\bar{\theta}$ in order to prove that $\Delta\left(1, \bar{S}, G_{0} / G_{1}\right)[\bar{\theta}]$ is a commutative ring. Let $i$ be the integer defined by $\rho(\zeta)=\zeta^{i}$. Since $i=n(\rho)$ according 
to Lemma 2.3, we obtain the congruence $u_{\rho} \theta \equiv \frac{1}{1-\zeta^{i}}\left(u_{\tau}^{i}-1\right) u_{\rho} \bmod$ $\Pi \Gamma_{w}$. The equalities $1-\zeta^{i}=(1-\zeta)\left(1+\zeta+\cdots+\zeta^{i-1}\right)$ and $u_{\tau}^{i}-1=\left(u_{\tau}-1\right)$ $\left(u_{\tau}^{i-1}+\cdots+1\right)$ in $\Gamma_{w}$ imply that $1-\zeta^{i} \equiv i(1-\zeta) \bmod \Pi \Gamma_{w}$ and $u_{\tau}^{i}-1$ $\equiv i\left(u_{\tau}-1\right) \bmod \Pi \Gamma_{w}$ since $\zeta \equiv 1 \bmod \Pi \Gamma_{w}$ and $u_{\tau} \equiv 1 \bmod \Pi \Gamma_{w} \cdot$ These congruences imply that $u_{\rho} \bar{\theta}=\bar{\theta} u_{\rho}$ in $\bar{\Gamma}_{w}$ and we conclude therefore that $\Delta\left(1, \bar{S}, G_{0} / G_{1}\right)[\bar{\theta}]$ is a commutative subring of $\bar{\Gamma}_{w}$.

In order to prove semi-simplicity, we first prove that $\Delta\left(1, \bar{S}, G_{0} / G_{1}\right)[\bar{\theta}]$ is a free (left) $\Delta\left(1, \bar{S}, G_{0} / G_{1}\right)$-module with free basis $\left\{1, \bar{\theta}, \ldots, \bar{\theta}^{p-1}\right\}$. The proof is by contradiction. So suppose that there exist elements $\bar{\delta}_{i}$ of $\Delta\left(1, \bar{S}, G_{0} / G_{1}\right)$ such that $\sum_{i=0}^{x} \bar{\delta}_{i} \bar{\theta}^{i}=\overline{0}$ with $\bar{\delta}_{x} \neq \overline{0}$ and $x \leq p-1$. Then $\sum_{i=0}^{x} \bar{\delta}_{i} \bar{\theta}^{i} \bar{\theta}^{(p-1-x)}=\overline{0} \quad$ so that we may consider an expression $\sum_{i=0}^{p-1} \bar{\delta}_{i} \bar{\theta}^{i}=\overline{0}$ where $\bar{\delta}_{p-1} \neq \overline{0}$. The method of proof shall be to contradict the assumption that $\bar{\delta}_{p-1}$ is non-zero. It is clear that we may choose representatives $\delta_{i}$ in $\Gamma_{w}$ of the residue classes $\bar{\delta}_{i}$ such that each $\delta_{i}$ is in $\Delta\left(g, S_{w}, G_{0}\right)$. Now the equality $\sum \bar{\delta}_{i} \bar{\theta}^{i}=\overline{0}$ implies that $\sum \delta_{i} \theta^{i}$ is in $\left(\Pi \Gamma_{w}\right) \cap \Delta\left(g, S_{w}, G_{0}\right)[\theta]$, and therefore $\sum \delta_{i} \theta^{i}$ is in $\Pi \Delta\left(g, S_{w}, G_{0}\right)[\theta]$ according to Lemma 2.16 A. Since $\Delta\left(g, S_{w}, G_{0}\right)[\theta]$ is generated as a left $\Delta\left(g, S_{w}, G_{0}\right)$-module by $\left\{1, \theta, \ldots, \theta^{p-1}\right\}$, it follows that $(1-\zeta)^{p-1} \sum \delta_{i} \theta^{i}$ is in $\Pi \Delta\left(g, S_{w}, G_{0}\right)$. Finally, the fact that $(1-\zeta)^{p-1} \sum_{i=0}^{p-2} \delta_{i} \theta^{i}$ is in $\Pi \Delta\left(g, S_{w}, G_{w}\right)$ implies that $(1-\zeta)^{p-1} \delta_{p-1} \theta^{p-1}=\delta_{p-1}\left(u_{\tau}-1\right)^{p-1}$ is in $\Pi \Delta\left(g, S_{w}, G_{0}\right)$. It remains to show that $\delta_{p-1}$ is in $\Pi \Gamma_{w}$. Consider a disjoint (left) coset decomposition $G_{0}=\cup \omega_{i} G_{1}$ of $G_{0}$ with respect to the subgroup $G_{1}$, and recall that $\Delta\left(g, S_{w}, G_{0}\right)$ is a free right $\Delta\left(1, S_{w}, G_{1}\right)$ module with free basis $\left\{u_{\omega_{i}}\right\}$. We may therefore consider a (unique) expression for $\delta_{p-1}$ of the form $\delta_{p-1}=\sum_{i} u_{\omega_{i}} \gamma_{i}$ with the $\gamma_{i}$ in $\Delta\left(1, S_{w}, G_{1}\right)$. The equality $\delta_{p-1}\left(u_{\tau}-1\right)^{p-1}=\sum u_{\omega_{i}} \gamma_{i}\left(u_{\tau}-1\right)^{p-1}$ together with the fact that $\delta_{p-1}\left(u_{\tau}-1\right)^{p-1}$ is in $\Pi \Delta\left(g, S_{w}, G_{0}\right)$ now implies that $\gamma_{i}\left(u_{\tau}-1\right)^{p-1}$ is in $\Pi \Delta(1$, $\left.S_{w}, G_{1}\right)$ for each $i$. The radical of $\Delta\left(1, S_{w}, G_{1}\right)$ is generated as a left ideal by $\Pi$ and $u_{\tau}-1$, and the residue class $\operatorname{ring} \Delta\left(1, S_{w}, G_{1}\right) / \operatorname{rad} \Delta\left(1, S_{w}, G_{1}\right)$ is isomorphic to $\bar{S}$. We may consider therefore for each $\gamma_{i}$ an element $s_{i}$ of $S$ and elements $\alpha_{i}$ and $\beta_{i}$ of $\Delta\left(1, S_{w}, G_{1}\right)$ such that $\gamma_{i}=s_{i}+\alpha_{i} \Pi+\beta_{i}\left(u_{\tau}-1\right)$. Then $\gamma_{i}\left(u_{\tau}-1\right)^{p-1}=s_{i}\left(u_{\tau}-1\right)^{p-1}+\alpha_{i} \Pi\left(u_{\tau}-1\right)^{p-1}+\beta_{i}\left(u_{\tau}-1\right)^{p}$. Since $\left(u_{\tau}-1\right)^{p}$ and $\gamma_{i}\left(u_{\tau}-1\right)^{p-1}$ are in $\Pi \Delta\left(1, S_{w}, G_{1}\right)$ it now follows that $s_{i}\left(u_{\tau}-1\right)^{p-1}$ is in $\Pi \Delta\left(1, S_{w}, G_{1}\right)$. Using the fact that $\Delta\left(1, S_{w}, G_{1}\right)$ is a free (left) $S_{w}$-module 
with free basis $\left\{u_{\tau^{i}}\right\}$ for $0 \leq i \leq p-1$ we may conclude that $s_{i}$ is in $\Pi S$ for each $i$, and therefore each $\gamma_{i}$ is in $\operatorname{rad} A\left(1, S_{w}, G_{1}\right)$. The element $u_{\tau}-1$ is in $\Pi \Delta\left(g, S_{w}, G_{1}\right)[\theta]$, so we obtain at last that $\delta_{p-1}$ is in $\Pi \Gamma_{w}$. Thus we have established that $\Delta\left(1, \bar{S}, G_{0} / G_{1}\right)[\bar{\theta}]$ is a free left $\Delta\left(1, \bar{S}, G_{0} / G_{1}\right)$-module with free basis $\left\{1, \bar{\theta}, \ldots, \bar{\theta}^{p-1}\right\}$.

Consider the polynomial ring $\Delta\left(1, \bar{S}, G_{0} / G_{1}\right)[Y]$ and form the residue class ring $\Delta\left(1, \bar{S}, G_{0} / G_{1}\right)[Y] /\left(Y^{p}-Y\right)$. Define a map $\varphi: \Delta\left(1, \bar{S}, G_{0} / G_{1}\right)[\bar{\theta}] \longrightarrow$ $\Delta\left(1, \bar{S}, G_{0} / G_{1}\right)[Y] /\left(Y^{p}-Y\right)$ in the following way. An element of $\Delta\left(1, \bar{S}, G_{0} / G_{1}\right)[\bar{\theta}]$ has a unique expression in the form $\sum_{i=1}^{p-1} \bar{\delta}_{i} \bar{\theta}^{i}$ with the $\bar{\delta}_{i}$ in $\Delta\left(1, S, G_{0} / G_{1}\right)$ according to the above. Define $\varphi\left(\sum \bar{\delta}_{i} \bar{\theta}^{i}\right)=\sum \bar{\delta}_{i} Y^{i}+\left(Y^{p}-Y\right)$. Cor. 2.10 implies that $\bar{\theta}^{p}=\bar{\theta}$, from which it follows that $\varphi$ is a monomorphism. It is easy to verify that $\varphi$ is in fact an $R$-algebra isomorphism.

Now we may establish the semi-simplicity of $\Delta\left(1, \bar{S}, G_{0} / G_{1}\right)[\bar{\theta}]$. Since the order of $G_{0} / G_{1}$ is relatively prime to the characteristic of $\bar{S}$, the group ring $\Delta\left(1, \bar{S}, G_{0} / G_{1}\right)$ is semi-simple. The polynomial $Y^{p}-Y$ factors into linear factors with no repeated roots in $\bar{S}[Y]$, namely $Y^{p}-Y=Y \prod_{i=1}^{p-1}\left(Y-\xi^{i}\right)$ where $\xi$ is a primitive $(p-1)^{s t}$ root of unity in $\bar{S}$ whose existence is guaranteed by the fact that $\bar{S}$ has characteristic $p$. For convenience of notation let $h_{0}(Y)=Y$ and $h_{i}(Y)=Y-\xi^{i}$ for $1 \leq i \leq p-1$. By the Chinese Remainder Theorem we have that the ring $\Delta\left(1, \bar{S}, G_{0} / G_{1}\right)[Y] /\left(Y^{p}-Y\right)$ is isomorphic to $\bigoplus_{i=0}^{p-1} \Delta\left(1, \bar{S}, G_{0} / G_{1}\right)[Y] /\left(h_{i}(Y)\right)$. Fach polynomial $h_{i}(Y)$ is linear so that each summand is isomorphic to $\Delta\left(1, \bar{S}, G_{0} / G_{1}\right)$. Therefore $\Delta\left(1, \bar{S}, G_{0} / G_{1}\right)[Y] /\left(Y^{p}-Y\right)$ is isomorphic to a direct sum of semi-simple rings and is therefore itself semi-simple. The fact that $\Delta\left(1, \bar{S}, G_{0} / G_{1}\right)[\bar{\theta}]$ is isomorphic to $\Delta\left(1, \bar{S}, G_{0} / G_{1}\right)$ $[Y] /\left(Y^{p}-Y\right)$ now implies that $\Delta\left(1, \bar{S}, G_{0} / G_{1}\right)[\bar{\theta}]$ is semi-simple.

Lemma $2.16 \mathrm{C}$. The residue class ring $\bar{\Gamma}_{w}$ is a finitely generated free left $\Delta\left(1, \bar{S}, G_{0} / G_{1}\right)[\bar{\theta}]$-module with free basis $\left\{u_{\sigma_{i}}\right\}$ where $G_{w} / G_{1}=\cup\left(G_{0} / G_{1}\right) \sigma_{i}$ is a disjoint right coset decomposition of $G_{w} / G_{1}$ with respect to the subgroup $G_{0} / G_{1}$.

Proof. It follows at once from Prop. 2.11 that $\bar{\Gamma}_{w}$ is generated as a right $\Delta\left(\bar{g}, \bar{S}, G_{w} / G_{1}\right)$-module by $\left\{1, \bar{\theta}, \ldots, \bar{\theta}^{p-1}\right\}$. Therefore an element $\lambda$ of $\bar{\Gamma}_{w}$ can be written in the form $\lambda=\sum \bar{\theta}^{i} \tilde{\delta}_{i}$ with the $\delta_{i}$ in $\Delta\left(\bar{g}, \bar{S}, G_{w} / G_{1}\right)$. Consider the elements $\sigma_{i}$ defined in the statement of the lemma. The 
crossed product $\Delta\left(\bar{g}, \bar{S}, G_{w} / G_{1}\right)$ is generated as a free (left) $\Delta\left(1, \bar{S}, G_{0} / G_{1}\right)$ module by the $\left\{u_{\sigma_{i}}\right\}$ (see Lemma 2.5 of [12]). Therefore each $\delta_{i}$ can be written in the form $\delta_{i}=\sum_{j} \gamma_{j}^{(i)} u_{\sigma_{j}}$ with the $\gamma_{j}^{(i)}$ in $\Delta\left(1, \bar{S}, G_{0} / G_{1}\right)$. Since $\lambda=$ $\sum_{i} \bar{\theta}^{i} \sum_{j} \gamma_{j}^{(i)} u_{\sigma_{j}}=\sum_{j}\left(\sum_{i} \bar{\theta}^{i} \gamma_{j}^{(i)}\right) u_{\sigma_{j}}$ we may conclude that the $\left\{u_{\sigma_{j}}\right\}$ generate $\bar{\Gamma}_{w}$ as a left $\Delta\left(1, \bar{S}, G_{0} / G_{1}\right)[\bar{\theta}]$-module.

It remains to show that the $\left\{u_{\sigma_{i}}\right\}$ are linearly independent over $\Delta\left(1, \bar{S}, G_{0} / G_{1}\right)[\bar{\theta}]$. So suppose that $\sum_{i=1} A_{i} u_{\sigma_{i}}=0$ for elements $A_{i}$ of $\Delta(1, \bar{S}$, $\left.G_{0} / G_{1}\right][\bar{\theta}]$. If the $A_{i}$ are not all zero, define $t\left(\left\{A_{i}\right\}\right)$ to be the largest integer $i$ such that $A_{i} \neq 0$; if $A_{i}=0$ for each $i$, define $t\left(\left\{A_{i}\right\}\right)=0$. The proof is by induction on $t\left(\left\{A_{i}\right\}\right)$. If $t\left(\left\{A_{i}\right\}\right)=1$, then $A_{1}=A_{1} u_{\sigma_{1}}\left(u_{\sigma_{1}}\right)^{-1}=0$ contradicting the assumption that $t\left(\left\{A_{i}\right\}\right)=1$. For the inductive step consider a set of elements $\left\{A_{i}\right\}$ of $\Delta\left(1, \bar{S}, G_{0} / G_{1}\right)[\bar{\theta}]$ such that $\sum A_{i} u_{\sigma_{i}}=0$ and $t\left(\left\{A_{i}\right\}\right)=t$. The induction hypothesis states that if $\left\{B_{i}\right\}$ is a set of elements of $\Delta\left(1, \bar{S}, G_{0} / G_{1}\right)[\bar{\theta}]$ such that $\sum B_{i} u_{\sigma_{i}}=0$ and $t\left(\left\{B_{i}\right\}\right)<t$, then $B_{i}=0$ for each $i$. Observe that $G_{w} / G_{0}$ is the Galois group of $\bar{S}$ over $\bar{R}$ and consider an element $\alpha$ of $\bar{S}$ such that $\bar{S}=\bar{R}(\alpha)$. The assumption that $\sum A_{i} u_{\sigma_{i}}=0$ implies that $0=\alpha\left(\sum A_{i} u_{\sigma_{i}}\right)-\left(\sum A_{i} u_{\sigma_{i}}\right) \sigma_{t}^{-1}(\alpha)=\sum\left(\alpha-\sigma_{i} \sigma_{t}^{-1}(\alpha)\right) A_{i} u_{\sigma_{i}}$. Since $\sigma_{i} \sigma_{t}^{-1}(\alpha)=\alpha$ if and only if $i=t$, we have that $t\left(\left\{\left(\alpha-\sigma_{i} \sigma_{t}^{-1}(\alpha)\right) A_{i}\right\}\right)<t$. Using the induction hypothesis we may now conclude that $A_{i}=0$ for $1 \leq i \leq t-1$. Therefore $A_{t} u_{\sigma_{t}}=0$ since $\sum A_{i} u_{\sigma_{i}}=0$ and we obtain that $A_{t}=A_{t} u_{\sigma_{t}}\left(u_{\sigma_{t}}\right)^{-1}=0$ contradicting the assumption that $A_{t} \neq 0$. We have established therefore that an equality $\sum A_{i} u_{\sigma_{\imath}}=0$ with the $A_{i}$ in $\Delta(1, \bar{S}$, $\left.G_{0} / G_{1}\right)[\bar{\theta}]$ implies that $A_{i}=0$ for each $i$.

The semi-simplicity of $\bar{\Gamma}_{w}$ now follows from that of its subring $\Delta(1, \bar{S}$, $\left.G_{0} / G_{1}\right)[\bar{\theta}]$.

Lemma 2.17. The ring $\bar{\Gamma}_{w}$ is a semi-simple ring.

Proof. For convenience of notation we shall denote the subring $\Delta(1, \bar{S}$, $\left.G_{0} / G_{1}\right)[\bar{\theta}]$ of $\bar{\Gamma}_{w}$ by $\bar{\Gamma}_{0}$ throughout the proof of this lemma. We shall make use of the fact that $\left(\operatorname{rad} \bar{\Gamma}_{w}\right) \cap \bar{\Gamma}_{0}$ is contained in $\operatorname{rad} \bar{\Gamma}_{0}$ (see Lemma 2.4 of [12]) .

The first step is to prove that $\operatorname{rad} \bar{\Gamma}_{w}=\left(\operatorname{rad} \bar{\Gamma}_{0}\right) \bar{\Gamma}_{w}$. Consider a disjoint right coset decomposition $G_{w} / G_{1}=\cup\left(G_{0} / G_{1}\right) \sigma_{i}$ of $G_{w} / G_{1}$ with respect to the subgroup $G_{0} / G_{1}$. According to Lemma 2.16 C, an element $\lambda$ of $\bar{\Gamma}_{w}$ 
can be written uniquely in the form $\lambda=\sum_{i=1} \lambda_{i} u_{\sigma_{i}}$ where the $\lambda_{i}$ are in $\bar{\Gamma}_{0}$. For $\lambda \neq 0$, define $t(\lambda)$ to be the largest integer $i$ for which $\lambda_{i} \neq 0$, and define $t(0)=0$. The proof is by induction on $t(\lambda)$. If $\lambda$ is an element of $\operatorname{rad} \bar{\Gamma}_{w}$ for which $t(\lambda)=1$, then $\lambda$ is of the form $\lambda=\lambda_{1} u_{\sigma_{1}}$ with $\lambda_{1}$ in $\bar{\Gamma}_{0}$ so that $\lambda_{1}=\lambda\left(u_{\sigma_{1}}\right)^{-1}$ is in $\left(\operatorname{rad} \bar{\Gamma}_{w}\right) \cap \bar{\Gamma}_{0}$ and hence in $\operatorname{rad} \bar{\Gamma}_{0}$ according to the remark at the beginning of the proof. Therefore $\lambda$ is in $\left(\operatorname{rad} \bar{\Gamma}_{0}\right) \bar{\Gamma}_{w} . \quad$ For the inductive step we assume that if $\gamma=\sum \gamma_{i} u_{\sigma_{i}}$ is an element of $\operatorname{rad} \bar{\Gamma}_{w}$ for which $t(\gamma)<t$ then each element $\gamma_{i}$ of $\bar{\Gamma}_{0}$ is in $\operatorname{rad} \bar{\Gamma}_{0}$. Now let $\lambda=\sum \lambda_{i} u_{\sigma_{i}}$ be an element of $\operatorname{rad} \bar{\Gamma}_{w}$ such that $t(\lambda)=t$. Recall that $G_{w} / G_{0}$ is the Galois group of $\bar{S}$ over $\bar{R}$, and consider an element $\alpha$ of $\bar{S}$ for which $\bar{S}=$ $\bar{R}(\alpha)$. In order to apply the induction hypothesis we form the element $\gamma=\alpha \lambda-\lambda \sigma_{t}^{-1}(\alpha)$ and observe that $\gamma=\Sigma\left(\alpha-\sigma_{i} \sigma_{t}^{-1}(\alpha)\right) \lambda_{i} u_{\sigma_{i}}$ is in $\operatorname{rad} \bar{\Gamma}_{w}$. Since $\sigma_{i} \sigma_{t}^{-1}(\alpha)=\alpha$ if and only if $i=t$, we may conclude that $\gamma=\sum_{i=1}^{t-1}(\alpha-$ $\left.\sigma_{i} \sigma_{t}^{-1}(\alpha)\right) \lambda_{i} u_{\sigma_{i}}$ is an element of $\operatorname{rad} \bar{\Gamma}_{w}$ for which $t(\gamma)<t$. Since $\alpha-\sigma_{i} \sigma_{t}^{-1}(\alpha)$ $=0$ for $1 \leq i \leq t-1$, the induction hypothesis now implies that $\lambda_{i}$ is in $\operatorname{rad} \bar{\Gamma}_{0}$ for $1 \leq i \leq t-1$. Therefore $\lambda_{t} u_{t}$ is in $\operatorname{rad} \bar{\Gamma}_{w}$, so that $\lambda_{t}=\lambda\left(u_{t}\right)^{-1}$ is in $\left(\operatorname{rad} \bar{\Gamma}_{w}\right) \cap \bar{\Gamma}_{0}$ and therefore in $\operatorname{rad} \bar{\Gamma}_{0}$. We have now established that $\operatorname{rad} \bar{\Gamma}_{w}$ is contained in $\left(\operatorname{rad} \bar{\Gamma}_{0}\right) \bar{\Gamma}_{w}$.

The ring $\bar{\Gamma}_{0}$ is semi-simple according to Lemma 2.16 B. Therefore rad $\bar{\Gamma}_{0}=(0)$ and we obtain that $\operatorname{rad} \bar{\Gamma}_{w}=\left(\operatorname{rad} \bar{\Gamma}_{0}\right) \bar{\Gamma}_{w}=(0)$. Since $\bar{\Gamma}_{w}$ is an Artin ring with zero radical we conclude that $\bar{\Gamma}_{w}$ is semi-simple.

Proposition 2.18. The ring $\Gamma_{w}$ is an hereditary order with radical $\Gamma_{w} \Pi$.

Proof. The fact that $\Gamma_{w} \Pi$ is contained in $\operatorname{rad} \Gamma_{w}$ (Lemma 2.14) together with the fact that $\Gamma_{w} / \Gamma_{w} \Pi$ is semi-simple (Lemma 2.17) implies that $\operatorname{rad} \Gamma_{w}=\Gamma_{w} \Pi$. It is easy to verify using the definition of crossed product that $\Gamma_{w} \Pi$ is a free left $\Gamma_{w}$-module. Therefore $\Gamma_{w}$ is an hereditary order according to the Corollary to Theorem 2.2 of [4]

In order to prove that $\Gamma_{w}$ is a maximal order it remains to show that $\Gamma_{w} \Pi$ is the unique maximal two-sided ideal of $\Gamma_{w}$, i.e. that $\bar{\Gamma}_{w}$ is a simple ring. Since $\bar{\Gamma}_{w}$ is a semi-simple ring (Lemma 2.17), its number of simple components is equal to the number of primitive orthogonal idempotents required to generate its center. We shall prove that the idempotents in the center of $\bar{\Gamma}_{w}$ are contained in $\bar{R}$ and thus conclude that $\bar{\Gamma}_{w}$ is simple. 
Lemma 2.19. The center of $\bar{\Gamma}_{w}$ is contained in the subring $\bar{S}[\bar{\theta}]$.

Proof. Once again we denote $\Delta\left(1, \bar{S}, G_{0} / G_{1}\right)[\bar{\theta}]$ by $\bar{\Gamma}_{0}$. We show first that the center $C\left(\bar{\Gamma}_{w}\right)$ of $\bar{\Gamma}_{w}$ is contained in the subring $\bar{\Gamma}_{0}$ of $\bar{\Gamma}_{w}$. Consider a disjoint right coset decomposition $G_{w} / G_{1}=\cup\left(G_{0} / G_{1}\right) \sigma_{i}$ of $G_{w} / G_{1}$ with respect to the subgroup $G_{0} / G_{1}$, with $\sigma_{1}=1$. Let $\delta$ denote a nonzero element of $C\left(\bar{\Gamma}_{w}\right)$. According to Lemma $2.16 \mathrm{C} \delta$ may be written uniquely in the form $\delta=\sum_{i=1}^{t} \delta_{i} u_{\sigma_{i}}$ with the $\delta_{i}$ in $\bar{\Gamma}_{0}$ and $\delta_{t} \neq 0$. Let $\alpha$ denote an element of $\bar{S}$ for which $\bar{S}=\bar{R}(\alpha)$. Since $\delta$ is in $C\left(\bar{\Gamma}_{w}\right)$ we must have $\alpha \delta=\delta \alpha$ so that

$$
\begin{gathered}
\alpha \delta_{1}+\left(\alpha \delta_{2}\right) u_{\sigma_{2}}+\cdots+\left(\alpha \delta_{t}\right) u_{\sigma_{t}} \\
=\alpha \delta_{1}+\left(\sigma_{2}(\alpha) \delta_{2}\right) u_{\sigma_{2}}+\cdots+\left(\sigma_{t}(\alpha) \delta_{t}\right) u_{\sigma_{t}} .
\end{gathered}
$$

Therefore $\alpha \delta_{i}=\sigma_{i}(\alpha) \delta_{i}$ for $1 \leq i \leq t$ because $\bar{\Gamma}_{w}$ is a free left $\bar{\Gamma}_{0}$-module with free basis $\left\{u_{\sigma_{i}}\right\}$ (see Lemma 2.16 C). Write each element $\delta_{i}$ of $\bar{\Gamma}_{0}$ in the form $\delta_{i}=\sum \lambda_{j}^{(i)} \bar{\theta}^{j}$ with the $\lambda_{j}^{(i)}$ in $\Delta\left(1, \bar{S}, G_{0} / G_{1}\right)$. The equalities $\alpha \delta_{i}=\sigma_{i}(\alpha) \delta_{i}$ imply that $\sum_{j} \alpha \lambda_{j}^{(i)} \bar{\theta}^{j}=\sum_{j} \sigma_{i}(\alpha) \lambda_{j}^{(i)} \bar{\theta}^{j}$ for each $i$. Using the fact that $\bar{\Gamma}_{0}$ is a free left $\Delta\left(1, \bar{S}, G_{0} / G_{1}\right)$-module with free basis $\left\{1, \bar{\theta}, \ldots, \bar{\theta}^{p-1}\right\}$ (see the proof of Lemma $2.16 \mathrm{~B})$ we conclude that $\alpha \lambda_{j}^{(i)}=\sigma_{i}(\alpha) \lambda_{j}^{(i)}$ for every $i$ and $j$. From the definition of crossed product it now follows that $\alpha=\sigma_{i}(\alpha)$ for each $i$. Since $\sigma_{i}(\alpha)=\alpha$ if and only if $i=1$, we obtain finally that $t=1$ and so $\delta$ is in $\bar{\Gamma}_{0}$.

It remains to prove that $\bar{\Gamma}_{0} \cap C\left(\bar{\Gamma}_{w}\right)$ is contained in $\bar{S}[\bar{\theta}]$. Consider an element $\delta$ of $\bar{\Gamma}_{0} \cap C\left(\bar{\Gamma}_{w}\right)$ and write $\delta$ in the form $\delta=\sum \lambda_{i} \bar{\theta}^{i}$ with the $\lambda_{i}$ in $\Delta\left(1, \bar{S}, G_{0} / G_{1}\right)$. Since $\delta$ is in $C\left(\bar{\Gamma}_{w}\right)$ we must have that $u_{\tau} \delta=\delta u_{\tau}$. Since $u_{\tau}$ commutes with $\bar{\theta}$ we now obtain the equality $\sum_{i} u_{\tau} \lambda_{i} \bar{\theta}^{i}=\sum_{i} \lambda_{i} u_{\tau} \bar{\theta}^{2}$. The fact that $\bar{\Gamma}_{0}$ is a free left $\Delta\left(1, \bar{S}, G_{0} / G_{1}\right)$-module with free basis $\left\{i, \bar{\theta}, \ldots, \bar{\theta}^{p-1}\right\}$ (Lemma $2.16 \mathrm{~B}$ ) implies that $u_{\tau} \lambda_{i}=\lambda_{i} u_{\tau}$ for each $i$. Write each element $\lambda_{i}$ of $\Delta\left(1, \bar{S}, G_{0} / G_{1}\right)$ in the form $\lambda_{i}=\sum_{\rho} \alpha_{\rho}^{(i)} u_{\rho}$ with the $\rho$ in $G_{0} / G_{1}$ and the $\alpha_{\rho}^{(i)}$ in $\bar{S}$. Then the equality $u_{\tau} \lambda_{i}=\lambda_{i} u_{\tau}$ implies that $\left.\sum_{\rho} \alpha_{\rho}^{(i)} u_{\tau \rho}=\sum_{\rho} \alpha_{\rho}^{(i)} u_{\tau} n^{\prime} \rho\right)_{\rho}$ where $n(\rho)$ is the integer defined modulo $(p)$ by $\rho \tau \rho^{-1}=\tau^{n(\rho)}$. According to Lemma $2.3 n(\rho)=1$ if and only if $\rho=1$. Therefore $\tau \rho=\tau^{n(\sigma)} \sigma$ for elements $\rho$ and $\sigma$ of $G_{0} / G_{1}$ if and only if $\rho=1$ and $\sigma=1$, from which it follows that each $\lambda_{i}$ is in $\bar{S}$, and hence that $\delta$ is in $\bar{S}[\bar{\theta}]$. 
Lemma 2.20. The idempotents in the center of $\bar{\Gamma}_{w}$ are contained in $\bar{R}$.

Proof. We first observe that the idempotents of $\bar{S}[\bar{\theta}]$ are present in $\bar{R}[\bar{\theta}]$. In the proof of Lemma $2.16 \mathrm{~B}$ it was shown that the $\operatorname{ring} \Delta(1, \bar{S}$, $\left.G_{0} / G_{1}\right)[\bar{\theta}]$ is a free left $\Delta\left(1, \bar{S}, G_{0} / G_{1}\right)$-module with free basis $\left\{1, \bar{\theta}, \ldots, \bar{\theta}^{p-1}\right\}$. From this it follows at once that $\bar{S}[\bar{\theta}]$ is a free $\bar{S}$-module with free basis $\left\{1, \bar{\theta}, \ldots, \bar{\theta}^{p-1}\right\}$ and that $\bar{R}[\bar{\theta}]$ is a free $\bar{R}$-module with free basis $\{1, \bar{\theta}, \ldots$, $\left.\bar{\theta}^{p-1}\right\}$. These observations imply that $\bar{S}[\bar{\theta}]$ is isomorphic to $\bar{S}[Y] /\left(Y^{p}-Y\right)$ and that $\bar{R}[\bar{\theta}]$ is isomorphic to $\bar{R}[Y] /\left(Y^{p}-Y\right)$. Recall from Lemma 2.16 $\mathrm{B}$ that $Y^{p}-Y=\prod_{i=0}^{p-1} h_{i}(Y)$ is a factorization of $Y^{p}-Y$ into linear factors in $\bar{R}[Y]$ where $h_{0}(Y)=Y$ and $h_{i}(Y)=Y-\xi^{i}$ for $1 \leq i \leq p-1$, and $\xi$ denotes a primitive $(p-1)^{s t}$ root of unity in $\bar{R}$. By the Chinese Remainder Theorem we obtain the isomorphisms $\bar{R}[Y] /\left(Y^{p}-Y\right) \approx \oplus \bar{R}[Y] /\left(h_{\imath}(Y)\right)$ and $\bar{S}[Y] /\left(Y^{p}-Y\right)$ $\approx \oplus \bar{S}[Y] /\left(h_{i}(Y)\right)$. The natural map of $\oplus \bar{R}[Y] /\left(h_{\imath}(Y)\right)$ into $\oplus \bar{S}[Y] /\left(h_{\imath}(Y)\right)$ maps the set of primitive orthogonal idempotents of $\oplus \bar{R}[Y] /\left(h_{i}(Y)\right)$ into such a system for $\oplus \bar{S}[Y] /\left(h_{i}(Y)\right)$. We conclude therefore that the idempotents of $\bar{S}[\bar{\theta}]$ are already present in $\bar{R}[\bar{\theta}]$.

In order to prove the lemma it suffices to show that the intersection $C\left(\bar{\Gamma}_{w}\right) \cap \bar{R}[\bar{\theta}]$ is contained in $\bar{R}$ since $C\left(\bar{\Gamma}_{w}\right)$ is contained in $\bar{S}[\bar{\theta}]$ according to Lemma 2.19. Let $\lambda$ denote a non-zero element of $C\left(\bar{\Gamma}_{w}\right) \cap \bar{R}[\bar{\theta}]$ and express $\lambda$ in the form $\lambda=\sum_{i=0}^{t} r_{i} \bar{\theta}^{i}$ where $0 \leq t \leq p-1$ and $r_{t} \neq 0$. To prove that $C\left(\bar{\Gamma}_{w}\right) \cap \bar{R}[\bar{\theta}]$ is contained in $\bar{R}$ we shall assume that $t>0$ and contradict the fact that $r_{t} \neq 0$.

Now according to Prop. 2.6 there exists an element $\sigma$ of $G_{w}$ such that $g\left(\tau^{n(\sigma)}, \sigma\right) \neq 1$. Therefore $g\left(\tau^{n(\sigma)}, \sigma\right)$ must be of the form $g\left(\tau^{n(\sigma)}, \sigma\right)=\zeta^{a}$ for the primitive $p^{t h}$ root of unity $\zeta$ and some integer a satisfying $1 \leq a \leq p-1$ (see Prop. 2.5). We shall now establish the equality $u_{\bar{\sigma}} \bar{\theta}=\left(\bar{\theta}+\frac{a}{n(\sigma)}\right) u_{\bar{\sigma}}$ where $\bar{\sigma}$ denotes the image of $\sigma$ under the natural map of $G_{w}$ onto $G_{w} / G_{1}$. From the definition of $\theta$ together with the fact that $g(\sigma, \tau)=1$ (see Prop. 2.5) we obtain the equality $u_{\sigma} \theta=\frac{1}{\left(1-\zeta^{\sigma}\right) \zeta^{a}}\left[\left(u_{\tau}^{n(\sigma)}-1\right)+\left(1-\zeta^{a}\right)\right] u_{\sigma} . \quad$ According to Lemma 2.3, $\zeta^{\sigma}=\zeta^{n(\sigma)}$. And the congruence $\zeta \equiv 1 \bmod \Pi S_{w}$ implies that $\zeta^{n(\sigma)-1}+\cdots+1 \equiv n(\sigma) \bmod \Pi S_{w}$. From these observations we obtain the congruence $u_{\sigma} \theta \equiv \frac{1}{n(\sigma)}\left[\frac{1}{1-\zeta}\left(u_{\tau}^{n(\sigma)}-1\right)+\frac{1-\zeta^{a}}{1-\zeta}\right] u_{\sigma} \bmod \Gamma_{w} \Pi$. Observe that $u_{\tau}^{n(\sigma)}-1=\left(u_{\tau}-1\right)\left(u_{\tau}^{n(\sigma)-1}+\cdots+1\right)$ so that $u_{\tau}^{n(\sigma)}-1 \equiv n(\sigma)\left(u_{\tau}-1\right)$ 
$\bmod \Gamma_{w} \Pi$ since $u_{\tau} \equiv 1 \bmod \Gamma_{w} \Pi$. This fact together with the congruence $\frac{1-\zeta^{a}}{1-\zeta} \equiv a \bmod \Gamma_{w} \Pi$ enables us to write $u_{\bar{\sigma}} \bar{\theta}=\left(\bar{\theta}+\frac{a}{n(\sigma)}\right) u_{\bar{\sigma}}$.

Since $\lambda$ is in $C\left(\bar{\Gamma}_{w}\right)$ we must have that $u_{\bar{\sigma}} \lambda=\lambda u_{\bar{\sigma}}$. From the above we may then obtain the equality $\sum_{i=0}^{t} r_{i} \bar{\theta}^{i}=\sum_{i=0}^{t} r_{i}\left(\bar{\theta}+\frac{a}{n(\sigma)}\right)^{i}$. Using the fact that $\bar{R}[\bar{\theta}]$ is a free $\bar{R}$-module with free basis $\left\{1, \bar{\theta}, \ldots, \bar{\theta}^{p-1}\right\}$ it follows from equating coefficients of $\bar{\theta}^{t-1}$ that $r_{t-1}=r_{t-1}+\frac{t a}{n(\sigma)} r_{t}$. Therefore $r_{t}=$ 0 , and this contradiction proves the lemma.

Corollary 2.21. The ring $\bar{\Gamma}_{W}$ is a simple ring.

Proof. The number of simple components of the semi-simple ring $\bar{\Gamma}_{w}$ is equal to the number of primitive orthogonal idempotents required to generate its center. Since the idempotent elements of $C\left(\bar{\Gamma}_{w}\right)$ are in $\bar{R}$ according to the lemma, we conclude that $\bar{\Gamma}_{w}$ is simple.

Proposition 2.22. The $R$-order $\Gamma_{w}$ in the central simple $k$-algebra $\Delta\left(g, L_{w}\right.$, $G_{w}$ ) has the following properties

i) $\Gamma_{w}$ is a maximal order with radical $\Gamma_{w} \Pi$

ii) $r\left(\Gamma_{w} / R\right)=r\left(S_{w} / R\right)$.

Proof. Prop. 2.18 together with Cor. 2.21 implies that $\Gamma_{w}$ is an hereditary order with unique maximal two-sided ideal. Therefore $\Gamma_{w}$ is a maximal order according to Thm. 2.3 of [4] .

Since $\Gamma_{w} \Pi$ is the radical of $\Gamma_{w}$ (see Lemma 2.17) and $\Pi$ is the prime element of $S_{w}$, it follows that the ramification index of $\Gamma_{w}$ over $R$ is equal to the ramification index of $S_{w}$ over $R$.

Now we prove the main result of this section.

Proposition 2.23. Let $k$ denote the quotient field of a complete discrete rank one valuation ring $R$ of unequal characteristic, and let $\Sigma$ denote a central simple $k$-algebra for which $\widetilde{\Sigma}$ is in $V(k)$. If $\widetilde{\Sigma}$ has Brauer number equal to the characteristic $p$ of $\bar{R}$, then a maximal order of $\Sigma$ is not equivalent to a crossed product over a tamely ramified extension of $R$.

Proof. Let $\Gamma$ denote a maximal order in a central simple algebra $\Sigma$ such that $\widetilde{\Sigma}$ satisfies the hypothesis of the theorem. If $\widetilde{\Sigma}$ has Brauer number $p$ there exists a maximal order $\Gamma_{w}$ equivalent to $\Gamma$ for which $r\left(\Gamma_{w} / R\right)$ is 
divisible by $p$ according to Prop. 2.22 . Therefore $r(\Gamma / R)$ is divisible by $p$, since ramification index is preserved under equivalence (Prop. 1.6).

However Props. 1.6 and 1.7 together imply that a maximal order equivalent to a crossed product over a tamely ramified extension of $R$ has ramification index relatively prime to the characteristic of $\bar{R}$.

3. The equicharacteristic case. The purpose of this section is to prove the assertion analagous to that of Prop. 2.23 in the case when $R$ is an equicharacteristic ring. If $R$ is an equicharacteristic ring of characteristic zero, then the Brauer number of $\widetilde{\Sigma}$ is relatively prime to the characteristic of $\bar{R}$ for every central simple $k$-algebra $\Sigma$; so for the purpose of this section we restrict our attention to the case of non-zero characteristic.

The following notation shall be in use throughout this section. The symbol $R$ shall denote an equicharacteristic complete discrete rank one valuation ring of non-zero characteristic, and $\Sigma$ shall denote a central simple algebra over the quotient field $k$ of $R$ for which $\Sigma$ is in $V(k)$ and such that the Brauer number of $\Sigma$ is equal to the characteristic $p$ of $\bar{R}$. Since $\Sigma$ is in $V(k)$, we may assume that $\Sigma$ is of the form $\Sigma=\Delta(f, L, G)$ for some unramified Galois extension $L$ of $k$.

Our object is to prove that under the assumption on the Brauer number of $\Sigma$, a maximal order in $\Sigma$ cannot be equivalent to a crossed product over a tamely ramified extension of $R$.

The method of proof is similar to that used in Section 2. We shall construct a central simple $k$-algebra $\Sigma_{w}=\Delta\left(g, L_{w}, G_{w}\right)$ equivalent to $\Sigma=$ $\Delta(f, L, G)$ with $L_{w}$ a wildly ramified extension of $k$ and with the 2-cocycle $g$ in $Z^{2}\left(G_{w}, U\left(S_{w}\right)\right)$, where $S_{w}$ denotes the integral closure of $R$ in $L_{w}$. As in Section 2 we then construct a maximal order $\Gamma_{w}$ in $\Sigma_{w}$ by adjoining an element $\theta$ of $\Sigma_{w}$ to the crossed product $\Delta\left(g, S_{w}, G_{w}\right)$ and prove that the ramification index $r\left(\Gamma_{w} / R\right)$ is equal to the characteristic of $\bar{R}$.

In order to construct the desired central simple $k$-algebra $\Sigma_{w}$ we first construct the extension $L_{w}$ of $k$. Let $S$ denote the integral closure of $R$ in $L$ and consider a prime element $\pi$ of $S$. It follows from Eisenstein's criterion that the polynomial $F(X)=X^{p}-\pi^{p-1} X-\pi$ of $S[X]$ is irreducible in $L[X]$, and we define $L_{w}$ to be the field obtained by adjoining a root $\Pi$ of $F(X)$ to $L$. 
Proposition 3.1. The chain of fields $L_{w} \supset L \supset k$ defined above has the following properties

i) if $\Pi$ denotes one root of $F(X)=X^{p}-\pi^{p-1} X-\pi$ then the other roots of $F(X)$ are given by $\Pi+\xi^{i} \pi$ for $1 \leq i \leq p-1$ where $\xi$ denotes a primitive $(p-1)^{\text {st }}$ root of unity in $R$

ii) $L_{w}$ is a Galois extension of $k$

iii) the extension $L_{w}$ of $L$ is wildly ramified of degree $p$, and $\Pi$ is a prime element of $L_{w}$.

Proof. Using the fact that $k$ has characteristic $p$, toghether with the fact that $F(\Pi)=0$, one may obtain that $F\left(\Pi+\xi^{i} \pi\right)=\left(\xi^{i p}-\xi^{i}\right) \pi^{p}$. But $\xi^{p}=\xi$ since $\xi$ is a $(p-1)^{s t}$ root of unity, and therefore $F\left(\Pi+\xi^{i} \pi\right)=0$ for $1 \leq i \leq p-1$.

It is clear from statement i) that $L_{w}$ is a Galois extension of $k$. The equality $\Pi^{p}=\pi\left(\pi^{p-2} \Pi+1\right)$ implies that $L_{w}$ is a wildly ramified inertial extension of $L$ of degree $p$ with prime element $\Pi$.

Henceforth $G_{w}$ shall denote the Galois group of $L_{w}$ over $k$, and $S_{w}$ the integral closure of $R$ in $L_{w}$. The next proposition describes the ramification groups of the extension $L_{w}$ of $k$.

Proposition 3.2. Let $G_{i}$ denote the $i^{\text {th }}$ ramification group of $L_{w}$ over $k$. Then

i) $G_{0}=G_{1}$

ii) $G_{1}$ is a cyclic group of order $p$

iii) $G_{1}$ is contained in the center of $G_{w}$

iv) $G_{1}=G_{\imath}$ and $G_{i+1}=$ (1) for $i=p-1$.

Proof. Statement i) is true because the extension $L_{w}$ of $k$ has no tame inertial part. Statement ii) follows at once from Prop. 3.1.

In order to prove that $G_{1}$ is contained in the center of $G_{w}$, consider the generator $\tau$ of $G_{1}$ defined by $\tau(\Pi)=\Pi+\xi \pi$ and an element $\sigma$ of $G_{w}$. Since $\tau$ leaves the elements of $S$ fixed, it follows that $\tau \sigma=\sigma \tau$ if and only if $\tau \sigma(\Pi)=\sigma \tau(\Pi)$. The conjugates of $\Pi$ relative to $k$ are precisely the conjugates of $\Pi$ relative to $L$ since the minimal polynomial $F(X)$ of $\Pi$ is in $k[X]$. Therefore $\sigma(\Pi)=\Pi$ or $\sigma(\Pi)=\Pi+\xi^{i} \pi$ for some integer $i$ such that $1 \leq i \leq p-1$ according to Prop. 3.1. When $\sigma(\Pi)=\Pi$ it is clear that 
$\tau \sigma(\Pi)=\sigma \tau(\Pi)$. So consider the case when $\sigma(\Pi)=\Pi+\xi^{i} \pi$. Using the fact that $\xi$ and $\pi$ are in $k$ it is easy to verify that $\tau \sigma(\Pi)$ and $\sigma \tau(\Pi)$ are both equal to $\Pi+\xi\left(1+\xi^{i-1}\right) \pi$. Therefore $\tau \sigma=\sigma \tau$ for all $\sigma$ in $G_{w}$, and hence $G_{1}$ is in the center of $G_{w}$.

Finally we observe that $p-1$ is a discontinuity in the sequence of ramification groups of $L_{w}$ over $k$. For if $\tau$ is the element of $G_{1}$ defined by $\tau(\Pi)=\Pi+\xi \pi$, then $\tau(\Pi)-\Pi=\xi \pi$ so that $\tau$ is in $G_{i}$ if and only if $1 \leq i \leq p-1$.

In order to define the central simple $k$-algebra $\Sigma_{w}=\Delta\left(g, L_{w}, G_{w}\right)$ it remains to define the 2-cocycle $g$ of $Z^{2}\left(G_{w}, U\left(L_{w}\right)\right)$. Now the assumption that the Brauer number of $\Sigma$ is $p$, where $\Sigma=\Delta(f, L, G)$, implies that the $p^{t h}$ power of the cohomology class $[f]$ is in the image of the natural map $H^{2}(G, U(S)) \longrightarrow H^{2}(G, U(L))$. There exists therefore a map $\phi: G \longrightarrow U(L)$ such that the 2-cocycle $h$ of $Z^{2}(G, U(L))$ defined by $h(\sigma, \tau)=f^{p}(\sigma, \tau) \phi(\sigma \tau) /$ $\phi(\sigma) \phi^{\sigma}(\tau)$ takes values in $U(S)$. Since $\phi(\sigma)$ is in $U(L)$ we may write $\phi(\sigma)=$ $\alpha_{\sigma} \pi^{\beta(\sigma)}$ where $\alpha_{\sigma}$ is in $U(S)$ and $\beta(\sigma)$ is an integer, and $\pi$ denotes the prime element of $S$. Define now the map $\phi_{w}: G_{w} \longrightarrow U\left(L_{w}\right)$ by $\phi_{w}(\sigma)=\Pi^{\beta(\bar{\sigma})}$ where $\bar{\sigma}$ denotes the image of $\sigma$ under the natural map of $G_{w}$ onto $G_{w} / G_{1}=G$, and $I I$ denotes the prime element of $S_{w}$. Define the element $g$ of $Z^{2}\left(G_{w}, U\left(L_{w}\right)\right)$ by

$$
g(\sigma, \tau)=f_{w}(\sigma, \tau) \phi_{w}(\sigma \tau) / \phi_{w}(\sigma) \phi_{w}^{\sigma}(\tau)
$$

where $f_{w}$ denotes the image of $f$ under the inflation map $Z^{2}(G, U(L)) \longrightarrow$ $Z^{2}\left(G_{w}, U\left(L_{w}\right)\right)$. The central simple $k$-algebra $\Delta\left(g, L_{w}, G_{w}\right)$ shall be denoted by $\Sigma_{w}$. The next three propositions present properties of the 2-cocycle $g$.

Proposition 3.3. The element $g$ defined above is in the image of the natural $\operatorname{map} Z^{2}\left(G_{w}, U\left(S_{w}\right)\right) \longrightarrow Z^{2}\left(G_{w}, U\left(L_{w}\right)\right)$.

Proof. Using the method of Prop. 2.4 one can verify that $g^{p}$ takes values in $U\left(S_{w}\right)$, from which it follows at once that $g$ is in the image of the natural map $Z^{2}\left(G_{w}, U\left(S_{w}\right)\right) \longrightarrow Z^{2}\left(G_{w}, U\left(L_{w}\right)\right)$.

Proposition 3.4. The 2-cocycle $g$ defined above has the following properties

i) $g(\sigma, \rho)=1$ for every $\sigma$ in $G_{w}$ and $\rho$ in $G_{1}$

ii) $g(\sigma, \rho) \equiv 1 \bmod \left(\Pi^{p-1}\right)$ for every $\sigma$ in $G_{1}$ and $\rho$ in $G_{w}$ 
iii) $\bar{g}$ is in the image of the inflation map $Z^{2}(G, U(\bar{S})) \longrightarrow Z^{2}\left(G_{w}, U(\bar{S})\right)$ where $\bar{g}$ denotes the image of $g$ under the natural map $Z^{2}\left(G_{w}, U\left(S_{w}\right)\right) \longrightarrow Z^{2}\left(G_{w}, U(\bar{S})\right)$.

Proof. As in the proof of Prop. 2.5 one can easily show that $\beta(1)=0$ where 1 denotes the identity element of $G$. Now let $\sigma$ denote an element of $G_{w}$ and $\rho$ an element of $G_{1}$. By the definition of $f_{w}$ we have $f_{w}(\sigma, \rho)$ $=f(\bar{\sigma}, \bar{\rho})=1$ where $\bar{\sigma}$ denotes the image of $\sigma$ under the natural map of $G_{w}$ onto $G$, so that $g(\sigma, \rho)=\phi_{w}(\sigma \rho) / \phi_{w}(\sigma) \phi_{w}^{\sigma}(\rho)=\Pi^{\beta(\bar{\sigma})} / \Pi^{\beta(\bar{\sigma})} \sigma\left(\Pi^{\beta(\overline{1})}\right)$. $\quad$ Since $\beta(\overline{1})=0$, it follows that $g(\sigma, \rho)=1$.

In order to prove statement ii), consider now an element $\sigma$ of $G_{1}$ and an element $\rho$ of $G_{w}$. The definition of $g$ together with the fact that $\beta(\overline{1})=0$ implies that $g(\sigma, \rho)=[\Pi / \sigma(\Pi)]^{\beta(\bar{\rho})}$. According to Prop. 3.1 we have $\sigma(\Pi)=\Pi$ or $\sigma(\Pi)=\Pi+\xi^{i} \pi$ where $\xi$ is a primitive $(p-1)^{s t}$ root of unity and $i$ is an integer satisfying $1 \leq i \leq p-1$. If $\sigma(\Pi)=\Pi$ it is clear that $g(\sigma, \rho)=1$; so consider the case when $\sigma(\Pi)=\Pi+\xi^{i} \pi$ for some $i$. Observe that $\pi=\Pi^{p}-\pi^{p-1} \Pi$ since $F(\Pi)=0$. Substituting this expression for $\pi$ one then obtains that $\sigma(\Pi) / \Pi=\left(\Pi+\xi^{i} \pi\right) / \Pi=1+\xi^{i}\left(\Pi^{p-1}-\pi^{p-1}\right)$, so that $\sigma(\Pi) / \Pi$ $\equiv 1 \bmod \left(\Pi^{p-1}\right)$. We may now conclude that $g(\sigma, \rho) \equiv 1 \bmod \left(\Pi^{p-1}\right)$.

For the proof of part iii) we first observe that $\bar{g}(\sigma, \rho)=\bar{g}(\rho, \sigma)=\overline{1}$ for every $\sigma$ in $G_{w}$ and $\rho$ in $G_{1}$ according to parts i) and ii) of this proposition. Using this observation it is easy to verify that the map $q: G \times G \longrightarrow U(\bar{S})$ defined by $q(\bar{\sigma}, \bar{\rho})=\bar{g}(\sigma, \rho)$ is an element of $Z^{2}(G, U(\bar{S}))$ in the preimage of $\bar{g}$.

Proposition 3.5. For each non-trivial element $\tau$ of $G_{1}$ there exists an element $\sigma$ of $G_{w}$ for which $g(\tau, \sigma) \equiv 1 \bmod \left(\Pi^{p-1}\right)$ and $g(\tau, \sigma) \neq 1 \bmod \left(\Pi^{p}\right)$.

Proof. Let $\beta: G \longrightarrow Z$ be the function used in the definition of the 2-cocycle $g$, and let $\bar{\sigma}$ denote the image of the element $\sigma$ of $G_{w}$ under the natural mapping of $G_{w}$ onto $G$. We shall show first that there exists an element $\sigma$ of $G_{w}$ such that $\beta(\bar{\sigma})$ is relatively prime to $p$. We shall then use the equality $g(\tau, \sigma)=\left(\Pi^{\beta(\tilde{\sigma})}\right) / \tau\left(\Pi^{\beta(\tilde{\sigma})}\right)$ to show that $g(\tau, \sigma) \neq 1 \bmod \left(\Pi^{p}\right)$.

We now show that there exists an element $\sigma$ of $G_{w}$ for which $\beta(\bar{\sigma}) \neq 0 \bmod (p)$. As in Prop. 2.6 the method of proof is to assume that $g(\bar{\sigma}) \equiv 0 \bmod (p)$ for every $\sigma$ in $G_{w}$ and then contradict the assumption on the Brauer number of $\widetilde{\Sigma}$, where $\Sigma$ denotes the central simple $k$-algebra $\Delta(f, L, G)$. If $\beta(\bar{\sigma}) \equiv 0 \bmod (p)$ for every $\sigma$ in $G_{w}$, then each integer $\beta(\bar{\sigma})$ 
may be written in the form $\beta(\bar{\sigma})=p r(\bar{\sigma})$ for some integer $r(\bar{\sigma})$. Define $\Psi: G \longrightarrow U(L)$ by $\Psi(\sigma)=\pi^{r(\sigma)}$. One can verify that the 2-cocycle $q$ of $Z^{2}(G, U(L))$ defined by $q(\sigma, \rho)=f(\sigma, \rho) \Psi(\sigma \rho) / \Psi(\sigma) \Psi^{\sigma}(\rho)$ is cohomologous to $f$ and takes values in $U(S)$, so that $[f]$ is in the image of the natural map $H^{2}(G, U(S)) \longrightarrow H^{2}(G, U(L))$. This contradicts the assumption that the Brauer number of $\widetilde{\Sigma}$ is $p$. Therefore there must exist an element $\sigma$ of $G_{w}$ for which $\beta(\bar{\sigma}) \neq 0 \bmod (p)$.

Finally we show that this $\sigma$ satisfies the assertion of the proposition. Part ii) of Prop. 3.4 implies that $g(\tau, \sigma) \equiv 1 \bmod \left(\Pi^{p-1}\right)$, so it remains to show that $\beta(\bar{\sigma}) \neq 0 \bmod (p)$ implies that $g(\tau, \sigma) \neq 1 \bmod \left(\Pi^{p}\right)$. We have already observed that $g(\tau, \sigma)=[\Pi / \tau(\Pi)]^{\beta(\bar{\sigma})}$ (see the proof of part ii) of Prop. 3.4). Let $u=\tau(\Pi) / \Pi$. Since $\tau$ was assumed to be non-trivial we must have that $\tau(\Pi)=\Pi+\xi^{i} \pi$ for some integer $i$. The equality $u=1$ $+\xi^{i}\left(\Pi^{p-1}-\pi^{p-1}\right)$ implies that $u \neq 1 \bmod \left(\Pi^{p}\right)$. It is easy to see that $u^{\beta(\bar{\sigma})} \neq 1 \bmod \left(\Pi^{p}\right)$ by writing $u^{\beta(\bar{\sigma})}-1$ in the form $u^{\beta(\bar{\sigma})}-1=(u-1)$ $\left(u^{\beta(\bar{\sigma})-1}+\cdots+1\right)$. For the fact that $\beta(\bar{\sigma})$ is relatively prime to $p$ implies that $u^{\beta(\bar{\sigma})-1}+\cdots+1$ is in $U\left(S_{w}\right)$, so that $u^{\beta(\bar{\sigma})} \equiv 1 \bmod \left(\Pi^{p}\right)$ if and only if $u \equiv 1 \bmod \left(\Pi^{p}\right)$. We may now conclude that $g(\tau, \sigma) \neq 1 \bmod$ $\left(\Pi^{p}\right)$.

Since the 2-cocycle $g$ is in $Z^{2}\left(G_{w}, U\left(S_{w}\right)\right)$ we may consider the crossed product $\Delta_{w}=\Delta\left(g, S_{w}, G_{w}\right)$. Observe, moreover, that $\Delta_{w}$ is an $R$-order in $\Sigma_{w}$. In order to construct the desired order $\Gamma_{w}$ containing $\Delta_{w}$, we first introduce some notation; throughout the rest of this section $\tau$ shall denote a fixed generator of $G_{1}$ and $\xi$ shall denote the primitive $(p-1)^{s t}$ root of unity defined by $\tau(\Pi)=\Pi+\xi \pi$. Consider the element $\theta$ of $\Sigma_{w}$ defined by $\theta=\frac{\Pi}{\pi}\left(u_{\tau}-1\right)$. Now $\Gamma_{w}$ is defined to be the ring obtained by adjoining the element $\theta$ to $\Delta_{w}$, i.e. $\Gamma_{w}=\Delta_{w}[\theta]$. Our main object is to prove that $\Gamma_{w}$ is a maximal order whose unique maximal two-sided ideal is generated by the prime element $\Pi$ of $S_{w}$.

The next two lemmas shall be useful in proving that $\Gamma_{w}$ is in fact an order over $R$ in $\Sigma_{w}$.

Lemma 3.6. For $1 \leq i \leq p-1$, let $a_{i}$ be the element of $\Delta\left(1, S_{w}, G_{1}\right)$ defined 
by $a_{i}=\frac{\Pi}{\pi}\left(\frac{u^{i}-1}{u^{2}}\right) u_{\tau}$ where $u$ is the element of $U\left(S_{w}\right)$ defined by $\tau(\Pi)=u \Pi$. Then $\left(\theta-a_{p-1}\right)\left(\theta-a_{p-2}\right) \cdots\left(\theta-a_{1}\right) \theta=0$.

Proof. Observe that each element $a_{i}$ of $\Delta\left(1, L_{w}, G_{1}\right)$ defined above is in fact an element of the crossed product $\Delta\left(1, S_{w}, G_{1}\right)$ (apply part iv) of Prop. 3.2) .

The first step is to prove inductively that $\left(\theta-a_{i}\right) \cdots\left(\theta-a_{1}\right) \theta=\left(\frac{\Pi}{\pi}\right)^{i+1}$ $\left(u_{\tau}-1\right)^{i+1}$. When $i=1$ we obtain by an easy computation the equalities $\left(\theta-a_{1}\right) \theta=\theta^{2}-a_{1} \theta=\left(\frac{\Pi}{\pi}\right)^{2}\left[\left(u u_{\tau}-1\right)-(u-1) u_{\tau}\right]\left(u_{\tau}-1\right)=\left(\frac{\Pi}{\pi}\right)^{2}\left(u_{\tau}-1\right)^{2} . \quad$ For the inductive step we assume that $\left(\theta-a_{i}\right) \cdots\left(\theta-a_{1}\right) \theta=\left(\frac{\Pi}{\pi}\right)^{i+1}\left(u_{\tau}-1\right)^{i+1}$. Then

$$
\begin{aligned}
& \left(\theta-a_{i+1}\right)\left(\theta-a_{i}\right) \cdots\left(\theta-a_{1}\right) \theta=\left(\theta-a_{i+1}\right)\left(\frac{\Pi}{\pi}\right)^{i+1}\left(u_{\tau}-1\right)^{i+1} \\
& \quad=\left(\frac{\Pi}{\pi}\right)^{i+2}\left[\left(u^{i+1} u_{\tau}-1\right)-\left(u^{i+1}-1\right) u_{\tau}\right]\left(u_{\tau}-1\right)^{i+1} \\
& \quad=\left(\frac{\Pi}{\pi}\right)^{i+2}\left(u_{\tau}-1\right)^{i+2}
\end{aligned}
$$

and this completes the inductive step.

From the above we may now conclude that $\left(\theta-a_{p-1}\right) \cdots\left(\theta-a_{1}\right) \theta=$ $\left(\frac{\Pi}{\pi}\right)^{p}\left(u_{\tau}-1\right)^{p}$. But $\left(u_{\tau}-1\right)^{p}=0$ since $\left(u_{\tau}\right)^{p}=1$ and $k$ has characteristic $p$. Therefore $\left(\theta-a_{p-1}\right) \cdots\left(\theta-a_{1}\right) \theta=0$.

Lemma 3.7. The ring $\Gamma_{W}$ is generated as both a left and right $\Delta\left(g, S_{w}\right.$, $\left.G_{w}\right)$-module by $\left\{1, \theta, \ldots, \theta^{p-1}\right\}$.

Proof. As in Prop. 2.11 we first prove that $\Gamma_{w}$ is generated as a right $\Delta_{w}$-module by powers of $\theta$. We shall obtain the inclusion $\Delta_{w}(\theta) \subset(1, \theta) \Delta_{w}$ by showing that $\left(\alpha_{\rho} u_{\rho}\right) \theta$ is contained in $(1, \theta) \Delta_{w}$ for every element $\rho$ of $G_{w}$ and $\alpha_{\rho}$ of $S_{w}$. Using the definition of $\theta$ one may obtain by a straightforward computation the equality

$$
\left(\alpha_{\rho} u_{\rho}\right) \theta=\frac{\Pi}{\pi}\left[u_{\tau} \tau^{-1}\left(\alpha_{\rho} v / g(\tau, \rho)\right)-v \alpha_{\rho}\right] u_{\rho}
$$

where $v$ is the element of $U\left(S_{w}\right)$ defined by $\rho(\Pi)=v \Pi$. From part ii) of Prop. 3. 4 we may obtain the congruence $\tau^{-1}(1 / g(\tau, \rho)) \equiv 1 \bmod \left(\Pi^{p-1}\right)$. The equality of ramification groups $G_{1}=G_{p-1}$ (see Prop. 3.2) implies that 
$\tau^{-1}\left(\alpha_{\rho} v\right) \equiv \alpha_{\rho} v \quad \bmod \left(\Pi^{p}\right)$. These two congruences together imply that $\tau^{-1}\left(\alpha_{\rho} v / g(\tau, \rho)\right) \equiv \alpha_{\rho} v \bmod \left(\Pi^{p-1}\right)$, so that $\tau^{-1}\left(\alpha_{\rho} v / g(\tau, \rho)\right)=\alpha_{\rho} v+s \Pi^{p-1}$ for some element $s$ of $S_{w}$. Substituting into the above expression for $\left(\alpha_{\rho} u_{\rho}\right) \theta$ we may then obtain the equality $\left(\alpha_{\rho} u_{\rho}\right) \theta=\left[\frac{\Pi}{\pi}\left(u_{\tau}-1\right) v \alpha_{\rho} u_{\rho}\right]+\left[\frac{\Pi}{\pi} u_{\tau} s \Pi^{p-1} u_{\rho}\right]$. The first summand is in $(\theta) \Delta_{w}$ and the second is in $\Delta_{w}$, so that $\left(\alpha_{\rho} u_{\rho}\right) \theta$ is in $(1, \theta) \Delta_{w}$.

It can now be proved inductively that $\Delta_{w}\left(\theta^{i}\right)$ is contained in $\left(\theta^{i-1}, \theta^{i}\right) \Delta_{w}$ for every positive integer $i$. Since $\theta$ satisfies an equation of degree $p$ over $\Delta\left(1, S_{w}, G_{1}\right)$ (see Lemma 3.6) we conclude finally that $\Gamma_{w}$ is generated as a right $\Delta_{w}$-module by $\left\{1, \theta, \ldots, \theta^{p-1}\right\} . \quad$ A similar argument shows that $\Gamma_{w}$ is generated as a left $\Delta_{w}$-module by $\left\{1, \theta, \ldots, \theta^{p-1}\right\}$.

Proposition 3.8. The ring $\Gamma_{w}$ is an order over $R$ in the central simple $k$ algebra $\Sigma_{w}$.

Proof. The proof of this assertion follows from Lemma 3.7 by an argument similar to that of Prop. 2.12.

We can prove that $\Gamma_{w}$ is an hereditary order by proving that its radical is $\Gamma_{w}$-projective.

Lemma 3.9. Let II denote the prime element of $S_{w}$. Then

i) $\Pi \Gamma_{w}=\Gamma_{w} \Pi$

ii) $\Pi$ is an element of rad $\Delta_{w}$

iii) $\operatorname{rad} \Delta_{w}=\left(\Pi, u_{\tau}-1\right) \Delta_{w}$

iv) $\Gamma_{w} \Pi \cap \Delta_{w}=\operatorname{rad} \Delta_{w}$.

Proof. Since $\Gamma_{w}$ is generated as a left $\Delta_{w}$-module by the elements $\left\{1, \theta, \ldots, \theta^{p-1}\right\}$ (Lemma 3.7) and $\Pi \Delta_{w}=\Delta_{w} \Pi$, it suffices to prove that $\theta \Pi$ is in $\Pi \Gamma_{w}$ in order to establish the inclusion $\Gamma_{w} \Pi \subset \Pi \Gamma_{w}$. The equality $\theta \Pi=\Pi \theta+\frac{\Pi}{\pi}(\tau(\Pi)-\Pi) u_{\tau}$ may be obtained by an easy computation. It is easy to verify that the element $\frac{1}{\pi}(\tau(\Pi)-\Pi)$ is in $S_{w}$ using the fact that there is a discontinuity in the sequence of ramification groups $G_{i}$ at $i=$ $p-1$ (Prop. 3.2). Therefore $\theta \Pi$ is in $\Pi \Gamma_{w}$ and $\Gamma_{w} \Pi$ is contained in $\Pi \Gamma_{w}$. A similar computation yields the opposite inclusion. Therefore $\Gamma_{w} \Pi=\Pi \Gamma_{w}$. By Lemma 1.4 of [12] we may now conclude that $\Pi$ is in $\operatorname{rad} \Gamma_{w}$. This completes the proof of statements i) and ii). 
The proof of part iii) is entirely similar to the proof of part iii) of Lemma 2.14 .

It remains to prove assertion iv). Lemma 2.13 implies that $\Gamma_{w} \Pi \cap \Delta_{w}$ is contained in $\mathrm{rad} \Delta_{w}$ since $\Pi$ is in $\mathrm{rad} \Gamma_{w}$ according to part ii). To obtain the opposite inclusion we make use of the fact that $\operatorname{rad} \Delta_{w}=$ (ח, $\left.u_{\tau}-1\right) \Delta_{w}$. The definition of $\theta$ implies that $u_{\tau}-1$ is in $\Pi^{p-1} \Gamma_{w}=$ $\Gamma_{w} \Pi^{p-1}$, from which it follows that $\operatorname{rad} \Delta_{w}$ is contained in $\Gamma_{w} \Pi$.

By Lemma 3.9 we may now form the residue class ring $\Gamma_{w} / \Gamma_{w} \Pi$ which shall henceforth be denoted by $\bar{\Gamma}_{w}$. An argument similar to that of Lemma 2.16 shows that $\bar{\Gamma}_{w}$ is $R$-algebra isomorphic to $\Delta(\bar{g}, \bar{S}, G)[\bar{\theta}]$ in a natural way where $\bar{\theta}$ denotes the residue class of $\theta$ modulo $\Gamma_{w} \Pi$.

In a manner similar to that of Section 2 , the semi-simplicity of $\bar{\Gamma}_{w}$ shall follow from that of its subring $\bar{S}[\bar{\theta}]$.

Lemma 3.10. For $1 \leq i \leq p-1$ let $a_{i}$ denote the element of $\Delta\left(1, S_{w}, G_{1}\right)$ defined in Lemma 3.6, and let $\bar{a}_{i}$ denote the image of $a_{i}$ in $\bar{\Gamma}_{w}$. Then $\bar{a}_{i}=\bar{\xi} i$ where $\xi$ denotes the primitive $(p-1)^{s t}$ root of unity defined by $\tau(\Pi)=\Pi+\xi \pi$.

Proof. From the definition of $a_{i}$ we obtain the equality $a_{i}=\frac{\Pi}{\pi}(u-1)$ $\left(\frac{u^{i-1}+\cdots+1}{u^{i}}\right) u_{\tau} . \quad$ Since $\frac{\Pi}{\pi}(u-1)=\frac{1}{\pi}(\tau(\Pi)-\Pi)=\xi$ we may write $a_{i}=\xi\left(\frac{u^{i-1}+\cdots+1}{u^{i}}\right) u_{\tau} . \quad$ The congruence $u_{\tau} \equiv 1 \bmod \Gamma_{w} \Pi$ holds because $\theta$ is in $\Gamma_{w}$. And $u \equiv 1 \bmod \Pi S_{w}$ since $G_{p-1}=G_{1}$ (see Prop. 3.2), so that $u^{i-1}+\cdots+1 \equiv i \bmod \Pi S_{w}$. Combining these observations we may now conclude that $\bar{a}_{\imath}=\bar{\xi} i$.

Lemma 3.11. The ring $\bar{\Gamma}_{w}$ is a semi-simple ring.

Proof. The first step is to observe that the subring $\bar{S}[\bar{\theta}]$ of $\bar{\Gamma}_{w}$ is a commutative semi-simple ring. Consider an element $\bar{\alpha}$ of $\bar{S}$ for which $\bar{S}=\bar{R}(\bar{\alpha})$. In order to establish the commutativity of $\bar{S}[\bar{\theta}]$ it suffices to prove that $\bar{\alpha} \bar{\theta}=\bar{\theta} \bar{\alpha}$. Now from the definition of $\theta$ we obtain the equality $\theta \alpha=\frac{\Pi}{\pi}\left(\tau(\alpha) u_{\tau}-\alpha\right)$ where $\alpha$ is an element of $S_{w}$ in the preimage of $\bar{\alpha} \cdot$ It is easy to see that the congruence $\tau(\alpha) \equiv \alpha \bmod \left(\Pi^{p}\right)$ implies that $\theta \alpha=\alpha \theta$ $\bmod \Gamma_{w} \Pi$. In order to prove that $\bar{S}[\bar{\theta}]$ is semi-simple we point out that a computation similar to that of Lemma 2.16 may be used to show that 
$\bar{S}[\bar{\theta}]$ is a free $\bar{S}$-module with free basis $\left\{1, \bar{\theta}, \ldots, \bar{\theta}^{p-1}\right\}$. From this it follows that $\bar{S}[\bar{\theta}]$ is isomorphic to the factor ring $\bar{S}[Y] /(H(Y))$ where $H(Y)=$ $\left(Y-\bar{a}_{p-1}\right) \cdots\left(Y-\bar{a}_{1}\right) Y$. Since $\bar{a}_{i}=\bar{\xi} i$ according to Lemma 3.10, we see that $\bar{a}_{i}=\bar{a}_{j}$ if and only if $i=j$, so that $H(Y)$ is a polynomial without repeated roots. We may now conclude from the Chinese Remainder Theorem that $\bar{S}[Y] /(H(Y))$ is isomorphic to a direct sum of $p$ copies of $\bar{S}$. This completes the proof that $\bar{S}[\bar{\theta}]$ is semi-simple.

An argument similar to that of Lemma $2.16 \mathrm{C}$ shows that $\bar{\Gamma}_{w}$ is a free left $\bar{S}[\bar{\theta}]$-module with free basis $\left\{u_{\sigma_{i}}\right\}$ where $\sigma_{i}$ ranges over the elements of $G$. Using this fact one can now establish by the method of Lemma 2.17 that $\bar{\Gamma}_{w}$ is a semi-simple ring.

Proposition 3.12. The ring $\Gamma_{w}$ is an hereditary $R$-order with radical $\Gamma_{w} \Pi$.

Proof. Since $\Pi$ is in $\operatorname{rad} \Gamma_{w}$ and $\Gamma_{w} / \Gamma_{w} \Pi$ is semi-simple it follows that $\operatorname{rad} \Gamma_{w}=\Gamma_{w} \Pi$. Thus $\Gamma_{w}$ is an hereditary order by the Corollary to Thm. 2.2 of $[4]$.

Lemma 3.13. The ideal $\Gamma_{w} \Pi$ is the unique maximal two-sided ideal of $\Gamma_{w}$.

Proof. In order to prove that $\Gamma_{w} \Pi$ is the unique maximal two-sided ideal of $\Gamma_{w}$ it suffices to prove that the semi-simple ring $\bar{\Gamma}_{w}$ is in fact a simple ring, and we do this by studying the idempotents in the center $C\left(\bar{\Gamma}_{w}\right)$ of $\bar{\Gamma}_{w}$.

An argument similar to that used in Lemma 2.19 shows that $C\left(\bar{\Gamma}_{w}\right)$ is contained in $\bar{S}[\bar{\theta}]$. It is easy to see that the idempotents of $\bar{S}[\bar{\theta}]$ are already present in $\bar{R}[\bar{\theta}]$. For consider the polynomial $H(Y)=(Y-$ $\left.\bar{a}_{p-1}\right) \cdots\left(Y-\bar{a}_{1}\right) Y$ of $\bar{S}[Y]$ and recall that the equation $H(Y)=0$ is satisfied by $\bar{\theta}$. Lemma 3.10 implies that the $\bar{a}_{i}$ are in $U(\bar{R})$, so that $H(Y)$ splits into $p$ (distinct) linear factors in $\bar{R}[Y]$. Using the Chinese Remainder Theorem once again, we conclude that $\bar{R}[\bar{\theta}]$ has precisely $p$ simple components. Since $\bar{R}[\bar{\theta}] \subset \bar{S}[\bar{\theta}]$ is an inclusion of commutative rings, we may now conclude that the idempotents of $\bar{S}[\bar{\theta}]$ are already present in $\bar{R}[\bar{\theta}]$.

We now use Prop. 3.5 to prove that the intersection $C\left(\bar{\Gamma}_{w}\right) \cap \bar{R}[\bar{\theta}]$ is contained in $\bar{R}$. The proof is by contradiction. Suppose that $\lambda=\sum r_{i} \bar{\theta}^{i}$ is a non-zero element of $C\left(\bar{\Gamma}_{w}\right) \cap \bar{R}[\bar{\theta}]$, where the $r_{\imath}$ are in $\bar{R}$ and $t$ is the largest integer for which $r_{t} \neq 0$. Prop. 3.5 implies that there exists an 
element $\sigma$ of $G_{w}$ such that $g(\tau, \sigma) \equiv 1 \bmod \left(\Pi^{p-1}\right)$ and $g(\tau, \sigma) \neq 1 \bmod \left(\Pi^{p}\right)$. Therefore we may write $g(\tau, \sigma)=1+w \Pi^{p-1}$ for some element $w$ of $U\left(S_{w}\right)$. Using the definition of $\theta$ together with the fact that $G_{1}$ is contained in the center of $G_{w}$ (Prop. 3.2) one may obtain by an easy computation the equality $u_{\bar{\sigma}} \bar{\theta}=(\bar{\theta}+\bar{w}) u_{\bar{\sigma}}$. Since $\lambda$ is in $C\left(\bar{\Gamma}_{w}\right)$ we must have $u_{\bar{\sigma}} \lambda=\lambda u_{\bar{\sigma}}$, so that $\sum r_{i} \bar{\theta}^{i}=\sum r_{i}(\bar{\theta}+\bar{w})^{i}$. This equality together with the fact that $\left\{1, \bar{\theta}, \ldots, \bar{\theta}^{p-1}\right\}$ is a free basis for $\bar{R}[\bar{\theta}]$ over $\bar{R}$ implies that $r_{t-1}=r_{t-1}+$ $t \bar{w} r_{t}$. Therefore $r_{t}=0$ and this contradiction establishes the desired inclusion.

Combining the above observations, we may conclude that the idempotent elements in the center of $\bar{\Gamma}_{w}$ are contained in $\bar{R}$. Therefore the semisimple ring $\bar{\Gamma}_{w}$ is a simple ring, and $\Gamma_{w} \Pi$ is the unique maximal two-sided ideal of $\Gamma_{w}$.

The arguments used in Props. 2.22 and 2.23 may now be used to prove the next two propositions.

Proposition 3.14. The R-order $\Gamma_{w}$ in the central simple k-algebra $\Sigma_{w}$ has the following properties

i) $\Gamma_{w}$ is a maximal order with radical $\Gamma_{w} \Pi$

ii) $\quad r\left(\Gamma_{w} / R\right)=r\left(S_{w} / R\right)$.

Proposition 3.15. Let $k$ denote the quotient field of a complete discrete rank one valuation ring $R$ which is an equicharacteristic ring of characteristic $p \neq 0$, and let $\Sigma$ denote a central simple k-algebra for which $\widetilde{\Sigma}$ is in $V(k)$. If $\widetilde{\Sigma}$ has Brauer number $p$, then a maximal order in $\Sigma$ is not equivalent to a crossed product over a tamely ramified extension of $R$.

Combining Propositions 2.23 and 3.15 we obtain the following theorem.

Theorem 3.16. Let $k$ denote the quotient field of a complete discrete rank one valuation ring $R$ such that the characteristic $p$ of $\bar{R}$ is non-zero, and let $\Sigma$ denote a central simple k-algebra for which $\widetilde{\Sigma}$ is in $V(k)$. If $\widetilde{\Sigma}$ has Brauer number $p$, then a maximal order in $\Sigma$ is not equivalent to a crossed product over a tamely ramified extension of $R$.

4. Maximal orders and the Brauer group. Let $k$ denote the quotient field of a complete discrete rank one valuation ring $R$. In this 
section we prove the main theorem of the paper, namely that a maximal order in a central simple $k$-algebra $\Sigma$ is equivalent to a crossed product over a tamely ramified extension of $R$ if and only if $\widetilde{\Sigma}$ is in $T(k)$. Both the necessity and sufficiency parts of the proof depend upon the main theorem on crossed products and maximal orders presented by the author in [11].

The following lemma shall be used to prove the sufficiency of the condition that $\widetilde{\Sigma}$ be in $T(k)$.

Lemma 4.1. Let $k$ denote the quotient field of a complete discrete rank one valuation ring $R$. Let $L$ be an unramified extension of $k$, and $\Sigma=\Delta(f, L, G) a$ crossed product for which $\widetilde{\Sigma}$ is in $T(k)$. Then there exists an extension $L_{t}$ of $L$ such that

i) $L_{t}$ is a tamely ramified Galois extension of $k$

ii) $\left[f_{t}\right]$ is in the image of the natural map $H^{2}\left(G_{t}, U\left(S_{t}\right)\right) \longrightarrow H^{2}\left(G_{t}, U\left(L_{t}\right)\right)$ where $S_{t}$ is the integral closure of $R$ in $L_{t}, G_{t}$ denotes the Galois group of $L_{t}$ over $k$, and $f_{t}$ is the image of $f$ under the inflation map $Z^{2}(G, U(L)) \longrightarrow Z^{2}\left(G_{t}, U\left(L_{t}\right)\right)$.

Proof. Let $e$ denote the Brauer number of $\widetilde{\Sigma}$. Since $e$ is relatively prime to $p$, it follows that the extension $L(\xi)$ of $L$ is unramified where $\xi$ denotes a primitive $e^{t h}$ root of unity. Next let $I I$ denote a root of the polynomial $X^{e}-\pi$ where $\pi$ is the prime element of $R$. Define $L_{t}=L(\xi, \Pi)$. It is easy to verify that the field $L_{t}$ is a tamely ramified Galois extension of $k$.

Let $S$ denote the integral closure of $R$ in $L$, and $S_{t}$ the integral closure of $R$ in $L_{t}$. It remains to construct a 2-cocycle $g$ of $Z^{2}\left(G_{t}, U\left(L_{t}\right)\right)$ such that $g$ is cohomologous to $f_{t}$ and such that $g$ is in the image of the natural map $Z^{2}\left(G_{t}, U\left(S_{t}\right)\right) \longrightarrow Z^{2}\left(G_{t}, U\left(L_{t}\right)\right)$. Since the image of $[f]$ in $H^{2}\left(G, Z^{+}\right)$has order $e$, it follows that there exists a map $\phi: G \longrightarrow U(L)$ such that the 2-cocycle $h$ of $Z^{2}(G, U(L))$ defined by $h(\sigma, \tau)=f^{e}(\sigma, \tau) \phi(\sigma \tau) / \phi(\sigma) \phi^{\sigma}(\tau)$ takes values in $U(S)$. Write the element $\phi(\sigma)$ of $U(L)$ in the form $\phi(\sigma)=\alpha_{\sigma} \pi^{\beta(\sigma)}$ where $\alpha_{\sigma}$ is in $U(S)$ and $\beta(\sigma)$ is an integer. Define the map $\phi_{t}: G_{t} \longrightarrow U\left(L_{t}\right)$ by $\phi_{t}(\sigma)=\Pi^{\beta(\bar{\sigma})}$ where $\bar{\sigma}$ denotes the image of $\sigma$ under the natural map of $G_{t}$ onto $G$. Define the element $g$ of $Z^{2}\left(G_{t}, U\left(L_{t}\right)\right)$ by $g(\sigma, \tau)=f_{t}(\sigma, \tau) \phi_{t}(\sigma \tau) /$ $\phi_{t}(\sigma) \phi_{t}^{\sigma}(\tau)$. Proceeding as in the proof of Prop. 2.4 one may easily verify 
that $g^{e}(\sigma, \tau)=h(\sigma, \tau) \alpha_{\sigma} \alpha_{\tau}^{\sigma} / \alpha_{\sigma \tau}$ from which it follows that the 2-cocycle $g$ takes values in $U\left(S_{t}\right)$.

Proposition 4.2. If $\Sigma$ is a central simple $k$-algebra for which $\widetilde{\Sigma}$ is in $T(k)$, then a maximal order in $\Sigma$ is equivalent to a crossed product over a tamely ramified extension of $R$.

Proof. Consider a representative $\Delta(f, L, G)$ of $\widetilde{\Sigma}$ where $L$ is an unramified extension of $k$. Since $\widetilde{\Sigma}$ is in $T(k)$ we may consider a field $L_{t}$ satisfying the conclusion of Lemma 4.1. Theorem 2.3 of [11] now implies that a maximal order in $\Delta(f, L, G)$ is equivalent to a crossed product over a tamely ramified extension of $R$.

In order to prove the main theorem in the other direction we first prove two propositions.

Proposition 4.3. If a central simple k-algebra $\sum$ is equivalent to a crossed product over a tamely ramified extension of $k$, then $\widetilde{\Sigma}$ is in $V(k)$.

Proof. According to the hypothesis we may consider a crossed product $\Delta(f, L, G)$ equivalent to $\Sigma$ for which the extension $L$ of $k$ is tamely ramified. Let $G_{I}$ denote the inertia group of $L$ over $k$ and let $f_{I}$ denote the image of $f$ under the restriction map $Z^{2}(G, U(L)) \longrightarrow Z^{2}\left(G_{I}, U(L)\right)$. We show first of all that $f$ may be replaced by a 2-cocycle $g$ whose restriction to $G_{I} \times G_{I}$ is normalized in the sense of cyclic groups. Consider a 2-cocycle $g_{I}$ in $Z^{2}\left(G_{I}, U(L)\right)$ which is cohomologous to $f_{I}$ and which is normalized in the sense of cyclic groups. Let $\phi_{I}: G_{I} \longrightarrow U(L)$ be a map for which $g_{I}(\sigma, \tau)=$ $f_{I}(\sigma, \tau) \phi_{I}(\sigma) \phi_{I}^{\sigma}(\tau) / \phi_{I}(\sigma \tau)$ for $\sigma$ and $\tau$ in $G_{I}$. Extend $\phi_{I}$ to a map $\phi: G \longrightarrow$ $U(L)$ by defining $\phi(\sigma)=\phi_{I}(\sigma)$ for $\sigma$ in $G_{I}$ and $\phi(\sigma)=1$ for $\sigma$ in $G-G_{I}$. Then the 2-cocycle $g$ of $Z^{2}(G, U(L))$ defined by $g(\sigma, \tau)=f(\sigma, \tau) \phi(\sigma) \phi^{\sigma}(\tau) / \phi(\sigma \tau)$ is cohomologous to $f$ and its restriction to $G_{I} \times G_{I}$ is normalized in the sense of cyclic groups. Since $[f]=[g]$ it follows that $\Delta(f, L, G)$ is $k$-algebra isomorphic to $\Delta(g, L, G)$.

Let $L_{I}$ denote the fixed field of $G_{I}$ and let a denote the element of $U\left(L_{I}\right)$ which defines the 2-cocycle $g_{I}$. Since $L$ is a tamely ramified inertial extension of $L_{I}$, the natural map $H^{2}\left(G_{I}, U(S)\right) \longrightarrow H^{2}\left(G_{I}, U(L)\right)$ is an epimorphism, where $S$ denotes the integral closure of $R$ in $L$ (see the proof of Cor. 2.4 of [11]). We may therefore assume that $a$ is in $U(U)$ where $U$ denotes the inertia ring of $L$ over $k$. 
We proceed to construct an unramified extension of $L$ which will give rise to an unramified splitting field of $\Sigma$. Let $e$ denote the order of $G_{I}$ and consider the element $\bar{a}$ of $U(\bar{U})$. Denote the order of $\bar{a}$ in $U(\bar{U}) /$ $[U(\bar{U})]^{e}$ by $e / m$. There exists an element $\bar{c}$ in $U(\bar{U})$ such that $\bar{a}=\bar{c}^{m}$, and the polynomial $X^{e / m}-\bar{c}$ is irreducible in $\bar{U}[X]$ (see the proof of Prop. 2.2 of [10]). Applying Hensel's lemma we may conclude that there exists an element $c$ in $U(U)$ for which $c^{m}=a$. Observe that the polynomial $P(X)=$ $X^{m}-c$ is irreducible in $L[X]$, and let $L(\alpha)$ be the field obtained by adjoining a root $\alpha$ of $P(X)$ to $L$. Since $L_{I}$ contains a primitive $e^{t h}$ root of unity, it is clear that $L(\alpha)$ is a Galois extension of $k$. It is easy to see that $L(\alpha)$ is an unramified extension of $L$. For let $S$ denote the integral closure of $R$ in $L$ and consider the ring $S[\alpha]$ where the brackets denote ring adjunction. According to Cor. 2 p. 66 of [7], the different $D$ of $S[\alpha]$ over $S$ is the principal ideal $\left(P^{\prime}(\alpha)\right)$. Since $P^{\prime}(X)=(e / m) X^{(e / m)-1}$ it follows that $D=S[\alpha]$ since $(p, e / m)=1$ and $\alpha$ is a unit in $S[\alpha]$. Hence $S[\alpha]$ is an unramified extension of $S$ and is therefore integrally closed in $L(\alpha)$.

We establish some notation which shall be used in the remainder of the proof. Let $G_{\alpha}$ denote the Galois group of $L(\alpha)$ over $k$; let $G_{I_{\alpha}}$ denote the inertia group of $L(\alpha)$ over $k$, and let $L_{I_{\alpha}}$ and $U_{\alpha}$ denote the inertia field and the inertia ring (respectively) of $L(\alpha)$ over $k$. Finally, denote by $g_{\alpha}$ the image of $g$ under the inflation map $Z^{2}(G, U(L)) \longrightarrow Z^{2}\left(G_{\alpha}, U(L(\alpha))\right)$ and observe that the crossed product $\Delta\left(g_{\alpha}, L(\alpha), G_{\alpha}\right)$ is equivalent to $\Delta(f, L, G)$.

The extension $L(\alpha)$ of $L$ has been constructed so that $g_{\alpha}$ shall be cohomologous to the trivial 2-cocycle on $G_{I_{\alpha}} \times G_{I_{\alpha}}$. For since the image of $G_{I_{\alpha}}$ under the natural map of $G_{\alpha}$ onto $G$ is $G_{I}$, it follows from the definition of the inflation map that $g_{I_{\alpha}}$ is defined by the element $a$ of $U(U)$ where $g_{I_{\alpha}}$ denotes the image of $g$ under the restriction map $Z^{2}\left(G_{\star}, U(L(\alpha))\right) \longrightarrow$ $Z^{2}\left(G_{I_{\alpha}}, U(L(\alpha))\right)$. It remains to show that $a \equiv 1 \bmod N(U(L(\alpha)))$ in $U\left(L I_{\alpha}\right) /$ $N(U(L(\alpha)))$. Since $\alpha^{e}=a$ and $\alpha$ is in $U\left(U_{\alpha}\right)$ we have that $N(\alpha)=a$, and therefore $g_{I_{\alpha}}$ is cohomologous to 1 .

Now we may complete the proof of the proposition. Since $H^{1}\left(G_{1}\right.$, $U(L(\alpha)))=(1)$ according to Prop. 2 p. 158 of [7] it follows from Prop. 5 p. 126 of [7] that the sequence

$$
(1) \longrightarrow H^{2}\left(G_{\alpha} / G_{I_{\alpha}}, U\left(L I_{\alpha}\right)\right) \stackrel{\text { inf }}{\longrightarrow} H^{2}\left(G_{\alpha}, U(L(\alpha))\right) \longrightarrow H^{2}\left(G I_{\alpha}, U(L(\alpha))\right)
$$

is exact. Therefore the fact that res $\left[g_{\alpha}\right]=[1]$ implies that there exists a 2- 
cocycle $h$ in $Z^{2}\left(G_{\alpha} / G_{I_{\alpha}}, U\left(L_{I_{\alpha}}\right)\right)$ such that inf $[h]=\left[g_{\alpha}\right]$. Now the crossed product $\Delta\left(h, L_{I_{\alpha}}, G_{\alpha} / G_{I_{\alpha}}\right)$ is equivalent to $\Delta\left(g_{\alpha}, L(\alpha), G_{\alpha}\right)$ and therefore to $\Sigma$. Since $L_{I_{\alpha}}$ is an unramified extension of $k$, we have proved that $\widetilde{\Sigma}$ is in $V(k)$.

Proposition 4.4. Let $k$ denote the quotient field of a complete discrete rank one valuation ring $R, L$ a finite Galois extension of $k$ with Galois group $G$, and $f$ an element of $Z^{2}(G, U(L))$. If a maximal order $\Gamma$ in $\Delta(f, L, G)$ is equivalent to a crossed product over a tamely ramified extension of $R$, then a maximal order $\Gamma_{x}$ in $\Delta\left(f^{x}, L, G\right)$ is equivalent to a crossed product over a tamely ramified extension of $R$ for every positive integer $x$.

Proof. Suppose that $\Gamma$ is equivalent to the crossed product $\Delta\left(g, S_{t}, G_{t}\right)$ where $S_{t}$ is a tamely ramified extension of $R$, and $G_{t}$ is the Galois group of the quotient field extension $L_{t}$ of $k$. The first step is to prove inductively that the central simple $k$-algebra $\Delta\left(f^{x}, L, G\right)$ is equivalent to $\Delta\left(g^{x}, L_{t}, G_{t}\right)$. For $x=1$ the assertion is trivial. So assume now that $\Delta\left(f^{x-1}, L, G\right)$ is equivalent to $\Delta\left(g^{x-1}, L_{t}, G_{t}\right)$. Now $\Delta\left(f^{x-1}, L, G\right) \otimes_{k} \Delta(f, L, G)$ is equivalent to $\Delta\left(f^{x}, L, G\right)$ and similarly $\Delta\left(g^{x-1}, L_{t}, G_{t}\right) \otimes_{k} \Delta\left(g, L_{t}, G_{t}\right)$ is equivalent to $\Delta\left(g^{x}, L_{t}, G_{t}\right)$ (see Thm. 8.5 A p. 86 of [1]). We may conclude therefore from the induction hypothesis that $\Delta\left(f^{x}, L, G\right)$ is equivalent to $\Delta\left(g^{x}, L_{t}, G_{t}\right)$.

A maximal order $\Gamma_{x}$ in $\Delta\left(f^{x}, L, G\right)$ is equivalent to a maximal order $\Omega_{x}$ in $\Delta\left(g^{x}, L_{t}, G_{t}\right)$ according to Lemma 2.1 of [11]. However, the fact that $S_{t}$ is a tamely ramified extension of $R$, together with the fact that $g$ is in $Z^{2}\left(G_{t}, U\left(S_{t}\right)\right)$ implies that $\Omega_{x}$ is equivalent to a crossed product over a tamely ramified extension of $R$ by Thm. 2.3 of [11] .

Now we may complete the proof of the main theorem.

TheOREm 4.5. Let $k$ denote the quotient field of a complete discrete rank one valuation ring $R$, and let $\Gamma$ be a maximal order in a central simple k-algebra $\Sigma$. Then $\Gamma$ is equivalent to a crossed product over a tamely ramified extension of $R$ if and only if the Brauer class $\widetilde{\Sigma}$ is in the subgroup $T(k)$ of $B(k)$.

Proof. If $\widetilde{\Sigma}$ is in $T(k)$, then a maximal order in $\Sigma$ is equivalent to a crossed product over a tamely ramified extension of $R$ according to Prop. 4.2.

On the other hand, assume now that $\Gamma$ is equivalent to a crossed product over a tamely ramified extension of $R$. Then $\widetilde{\Sigma}$ is in $V(k)$ according to Prop. 4.3, so that $\widetilde{\Sigma}$ may be represented by a crossed product 
$\Delta(f, L, G)$ where $L$ is an unramified extension of $k$. We prove by contradiction that the Brauer number $n$ of $\widetilde{\Sigma}$ must be relatively prime to $p$. The assertion is trivial when $\bar{R}$ has characteristic zero. We assume therefore that $n$ is divisible by char $\bar{R}=p \neq 0$, and write $n$ in the form $n=m p^{t}$ where $m$ is relatively prime to $p$ and $t \geq 1$. Consider the central simple $k$-algebra $\Sigma_{n / p}=\Delta\left(f^{n / p}, L, G\right)$ and observe that the Brauer number of $\widetilde{\Sigma}_{n / p}$ is $p$. If a maximal order in $\Delta(f, L, G)$ were equivalent to a crossed product over a tamely ramified extension of $R$, then a maximal order $\Omega$ in $\Delta\left(f^{n / p}, L, G\right)$ would be equivalent to a crossed product over a tamely ramified extension of $R$ according to Prop. 4.4. But $\Omega$ cannot be equivalent to such a crossed product because the Brauer number of $\widetilde{\Sigma}_{n / p}$ is $p$ (see Thm. 3.16). This contradiction completes the proof of the theorem.

COROllary 4.6. Let $k$ denote the quotient field of a complete discrete rank one valuation ring $R$ whose residue class field $\bar{R}$ is perfect, and let $\Gamma$ denote a maximal order in a central simple $k$-algebra $\Sigma$. Then the following statements are equivalent

i) $\Gamma$ is equivalent to a crossed product over a tamely ramified extension of $R$

ii) $\Gamma$ is equivalent to a crossed product

iii) the Brauer number of $\widetilde{\Sigma}$ is relatively prime to the characteristic of $\bar{R}$.

Proof. The equivalence of i) and ii) follows from Theorem 2 of [6] since a maximal order is hereditary and $\bar{R}$ is perfect. The equivalence of $i$ ) and iii) follows from the theorem.

COROllary 4.7. Let $R$ denote a complete discrete rank one valuation ring. If $R$ is an equicharacteristic ring of characteristic zero, then every maximal order over $R$ is equivalent to a crossed product over a tamely ramified extension of $R$.

Proof. This assertion follows immediately from Thm. 4.5 since $T(k)=$ $B(k)$ when $R$ is an equicharacteristic ring of characteristic zero.

COROLlary 4.8. There exist maximal orders which are not equivalent to crossed products.

Proof. Let $R$ denote the ring of $p$-adic integers $Z_{p}$, and $k$ the quotient field of $R$. In Remark 1.5 we observed that $T(k)$ is properly contained in $V(k)$. Since $\bar{R}$ is perfect, it follows from Cor. 4.6 that not every maximal order is equivalent to a crossed product. 


\section{REFERENCES}

[1] E. Artin, C. Nesbitt and R. Thrall, Rings with Minimum Condition. Michigan, (1955).

[2] E. Artin and J. Tate, Class field theory. Princeton notes, 1951. distributed by Harvard Univ.

[ 3 ] M. Auslander and O. Goldman, The Brauer group of a commutative ring, Trans. Amer. Math. Soc. Vol. 97 (1960), pp. 367-409.

[4] M. Auslander and O. Goldman, Maximal orders, Trans. Amer. Math. Soc. Vol. 97 (1960), pp. 1-24.

[ 5 ] M. Harada, Hereditary orders, Trans. Amer. Math. Soc. Vol. 107 (1963), pp. 273-290.

[6] M. Harada, Some criteria for hereditarity of crossed products, Osaka J. Math. Vol. 1 (1964), pp. $69-80$

[ 7 ] J.P. Serre, Corps Locaux, Paris, Hermann, (1962).

[8] B.L. van der Waerden, Modern Algebra, Vol. 1, Ungar, (1953).

[9] E. Weiss, Algebraic Number Theory, McGraw-Hill Co., (1963).

[10] S. Williamson, Crossed products and hereditary orders, Nagoya Math. J. Vol. 23 (1963), pp. $103-120$.

[11] S. Williamson, Crossed products and maximal orders, Nagoya Math. J. Vol. 25 (1965), pp. $165-174$.

[12] S. Williamson, Crossed products and ramification, Nagoya Math. J. Vol. 28 (1966) pp. 85111.

[13] A. Brumer, The structure of hereditary orders, Ph.D. Thesis, Princeton Univ., (1963).

Regis College

Weston, Massachusetts 